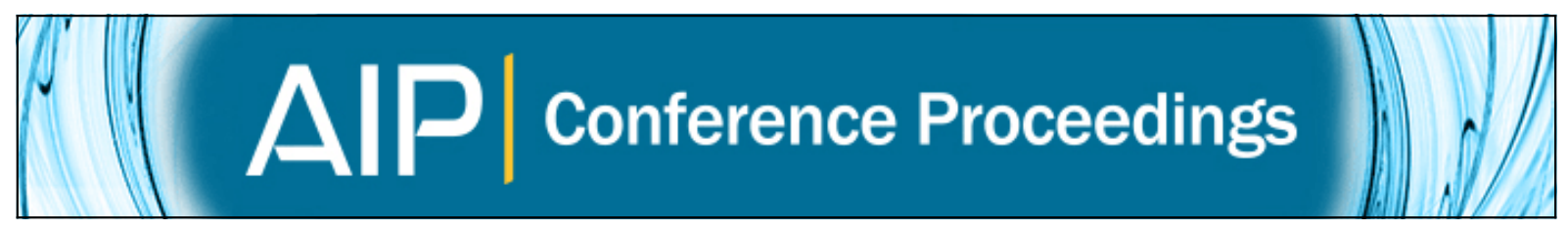

\title{
Prospects for polarized foreground removal
}

J. Dunkley, A. Amblard, C. Baccigalupi, M. Betoule, D. Chuss, A. Cooray, J. Delabrouille, C. Dickinson, G. Dobler , J. Dotson, H. K. Eriksen, D. Finkbeiner, D. Fixsen, P. Fosalba, A. Fraisse, C. Hirata, A. Kogut, J. Kristiansen, C. Lawrence, A. M. Magalhães, M. A. Miville-Deschenes, S. Meyer, A. Miller, S. K. Naess, L. Page, H. V. Peiris, N. Phillips, E. Pierpaoli, G. Rocha, J. E. Vaillancourt, and L. Verde

Citation: AIP Conference Proceedings 1141, 222 (2009); doi: 10.1063/1.3160888

View online: http://dx.doi.org/10.1063/1.3160888

View Table of Contents: http://scitation.aip.org/content/aip/proceeding/aipcp/1141?ver=pdfcov

Published by the AIP Publishing

\section{Articles you may be interested in}

Optical design of Argonne/KICP detectors for CMB polarization

AIP Conf. Proc. 1185, 487 (2009); 10.1063/1.3292384

Foreground Science Knowledge and Prospects

AIP Conf. Proc. 1141, 265 (2009); 10.1063/1.3160889

Polarized foregrounds power spectra vs CMB

AIP Conf. Proc. 609, 84 (2002); 10.1063/1.1471828

CMB polarization: Scientific case and data analysis issues

AIP Conf. Proc. 609, 78 (2002); 10.1063/1.1471827

Galactic synchrotron foreground and the CMB polarization measurements

AIP Conf. Proc. 609, 66 (2002); 10.1063/1.1471825 


\title{
Prospects for polarized foreground removal
}

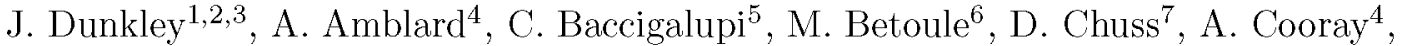 \\ J. Delabrouille ${ }^{6}$, C. Dickinson ${ }^{8,9}$, G. Dobler ${ }^{10}$, J. Dotson ${ }^{11}$, H. K. Eriksen ${ }^{12}$, \\ D. Finkbeiner ${ }^{10}$, D. Fixsen ${ }^{7}$, P. Fosalba ${ }^{13}$, A. Fraisse ${ }^{3}$, C. Hirata ${ }^{14}$, A. Kogut ${ }^{7}$, \\ J. Kristiansen ${ }^{12}$, C. Lawrence ${ }^{9}$, A. M. Magalhães ${ }^{15}$, M. A. Miville-Deschenes ${ }^{16}$, \\ S. Meyer ${ }^{17}$, A. Miller ${ }^{18}$, S. K. Naess ${ }^{12}$, L. Page ${ }^{2}$, H. V. Peiris ${ }^{19}$, N. Phillips ${ }^{7}$, \\ E. Pierpaoli ${ }^{20}$, G. Rocha ${ }^{8}$, J. E. Vaillancourt ${ }^{21}$, L. Verde $^{22}$
}

\begin{abstract}
In this report we discuss the impact of polarized foregrounds on a future CMBPol satellite mission. We review our current knowledge of Galactic polarized emission at microwave frequencies, including synchrotron and thermal dust emission. We use existing data and our understanding of the physical behavior of the sources of foreground emission to generate sky templates, and start to assess how well primordial gravitational wave signals can be separated from foreground contaminants for a CMBPol mission. At the estimated foreground minimum of $\sim 100 \mathrm{GHz}$, the polarized foregrounds are expected to be lower than a primordial polarization signal with tensor-to-scalar ratio $r=0.01$, in a small patch $(\sim 1 \%)$ of the sky known to have low Galactic emission. Over $75 \%$ of the sky we expect the foreground amplitude to exceed the primordial signal by about a factor of eight at the foreground minimum and on scales of two degrees. Only on the largest scales does the polarized foreground amplitude exceed the primordial signal by a larger factor of about 20 . The prospects for detecting an $r=0.01$ signal including degree-scale measurements appear promising, with $5 \sigma_{r} \sim 0.003$ forecast from multiple methods. A mission that observes a range of scales offers better prospects from the foregrounds perspective than one targeting only the lowest few multipoles. We begin to explore how optimizing the composition of frequency channels in the focal plane can maximize our ability to perform component separation, with a range of typically $40 \lesssim \nu \lesssim 300 \mathrm{GHz}$ preferred for ten channels. Foreground cleaning methods are already in place to tackle a CMBPol mission data set, and further investigation of the optimization and detectability of the primordial signal will be useful for mission design.
\end{abstract}

$\dagger$ j.dunkley@physics.ox.ac.uk

CP1141, CMB Polarization Workshop: Theory and Foregrounds, CMBPol Mission Concept Study edited by S. Dodelson, D. Baumann, A. Cooray, J. Dunkley, A. A. Fraisse, M. G. Jackson, A. Kogut, L. M. Krauss, K. M. Smith, and M. Zaldarriaga O 2009 American Institute of Physics 978-0-7354-0678-0/09/\$25.00 
1 Astrophysics, University of Oxford, Keble Road, Oxford, OX1 3RH, UK

${ }^{2}$ Department of Physics, Princeton University, Princeton, NJ 08540, USA

${ }^{3}$ Department of Astrophysical Sciences, Princeton, NJ 08540, USA

${ }^{4}$ Center for Cosmology, University of California, Irvine, CA 92697, USA

${ }^{5}$ SISSA, Via Beirut, Trieste 34014, Italy

${ }^{6}$ Laboratoire AstroParticule et Cosmologie, UMR 7164, CNRS and Observatoire de Paris, 10, 75205 Paris Cedex 13, France

${ }^{7}$ Code 665, NASA/Goddard Space Flight Center, Greenbelt, MD 20771, USA

${ }^{8}$ Infrared Processing 85 Analysis Center, California Institute of Technology, MC 220-6, Pasadena, CA 91125, USA

${ }^{9}$ NASA Jet Propulsion Laboratory, 4800 Oak Grove Drive, Pasadena, CA 91109, USA

${ }^{10}$ Harvard-Smithsonian Center for Astrophysics, 60 Garden St. - MS 51, Cambridge, MA 02138, USA

11 NASA Ames Research Center, MS 240-2, Moffett Field, CA 94035 USA

12 Institute of Theoretical Astrophysics, University of Oslo, P.O. Box 1029 Blindern, N-0315 Oslo, Norway

${ }^{13}$ Institut de Ciencies de l'Espai, IEEC-CSIC, F. de Ciencies, Torre C5 par-2, Barcelona 08193, Spain

14 Division of Physics, Mathematics, \& Astronomy, California Institute of Technology, MC 130-33, Pasadena, CA 91125, USA

${ }^{15}$ IAG, Universidade de São Paulo, Rua do Matão 1226, São Paulo 05508-900, Brazil

${ }^{16}$ Institut d'Astrophysique Spatiale, Universite Paris XI, Orsay, 91405, France

17 Kavli Institute for Cosmological Physics, University of Chicago, Chicago, IL 60637, USA

18 Physics Department, Columbia University, New York, NY 10027, USA

${ }^{19}$ Institute of Astronomy, University of Cambridge, Cambridge, CB3 OHA, UK

${ }^{20}$ University of Southern California, Los Angeles, CA, 90089-0484, USA

21 Division of Physics, Mathematics, 8 Astronomy, California Institute of Technology, MC 320-47, Pasadena, CA 91125, USA

${ }^{22}$ ICREA 8 Institute of Space Sciences (IEEC-CSIC), Barcelona, Spain 


\section{Introduction}

Measurements of the Cosmic Microwave Background (CMB) temperature anisotropy have led to the establishment of a standard $\Lambda$ CDM cosmological model for the universe (Miller et al. 1999; Lee et al. 2001; Netterfield et al. 2002; Halverson et al. 2002; Pearson et al. 2003; Scott et al. 2003; Spergel et al. 2003, 2007; Dunkley et al. 2008a). The polarization anisotropy, two orders of magnitude smaller, was measured for the first time in 2002 by DASI (Kovac et al. 2002; Leitch et al. 2002) and confirmed by the ground and balloon-based experiments CBI, CAPMAP, Boomerang, and QuAD (Readhead et al. 2004; Barkats et al. 2005; Montroy et al. 2006; P. Ade et al. 2007; Pryke et al. 2008). The first all-sky observations by the WMAP satellite (Page et al. 2007; Hinshaw et al. 2008), with a measurement of the power in the curl-free polarized 'E-mode', have provided a cross-check of the cosmological model, led to improved constraints on cosmological parameters, and a measurement of the optical depth to the reionization of the universe (Page et al. 2007; Hinshaw et al. 2008).

The next challenge of $\mathrm{CMB}$ observation is to test the inflationary scenario for the early universe, by looking for the signature of primordial gravitational waves. They are predicted to leave a distinct divergence-free, or 'B-mode', pattern in the large-scale CMB polarization anisotropy that may be observable with a future experiment (see Baumann et al. (2008b) for details). This signal is already constrained to be over an order of magnitude smaller than the observed E-mode signal arising from scalar fluctuations, from ground and space-based observations (Page et al. 2007; Hinshaw et al. 2008; Pryke et al. 2008). The Planck satellite, due for launch 2009, is projected to reach limits on the ratio of power in primordial tensor to scalar fluctuations $r$, of $r \sim 0.1$ (The Planck Collaboration 2006), while ground and balloon-based experiments currently observing or being commissioned are designed to reach below $r=0.1$. These include EBEX, Clover, QUIET, and SPIDER (see e.g., Oxley et al. (2004); Taylor (2006); Samtleben (2008); Crill et al. (2008) for descriptions).

This document is part of a study focusing on the capabilities of a future satellite mission targeting limits of $r=0.01$ or lower, known as CMBPol. Here we address the issue of polarized foreground emission from the Galaxy, which on average dominates the primordial B-mode over the whole sky. Establishing how well we can realistically hope to extract the primordial signal, and how this influences mission design, has been addressed in a number of previous studies (e.g., Amarie et al. (2005); Verde et al. (2006); Bock et al. (2006, 2008)). In companion documents the theoretical case for inflation and its predictions are presented (Baumann et al. 2008b), with weak lensing effects studied in Smith et al. (2008), and reionization prospects in Zaldarriaga et al. (2008). The Galactic science case is presented in Fraisse et al. (2008), and Baumann et al. (2008a) provide a summary.

The document is structured as follows. In $\S 2$ we review our current understanding of polarized foreground emission, and discuss predictions and models for the emission, with estimates of largescale polarization maps and power spectra. In $\S 3$ we describe various methods that are used to estimate polarized CMB maps, and in $\S 4$ apply these and Fisher matrix methods to begin to forecast limits for a specific template mission, and test optimal allocation of channels among frequencies. We also describe future tests, and summarize in $\S 5$.

\section{Knowledge of polarized foregrounds}

In this section we focus on observations of polarized foregrounds relevant to the microwave regime, concentrating on large-scale diffuse emission. The discussion is organized by physical emission mechanism, and outlines how our understanding of the physics of the interstellar medium is used to construct two-dimensional emission templates on the sky. This is an inherently simpler problem than a three-dimensional model. Issues that remain open for investigation include: possible spatial and frequency variation of spectral indices, the number of parameters required for a sufficiently sophisticated model to describe and subtract foregrounds at the level required if $r<0.01$, and methods 


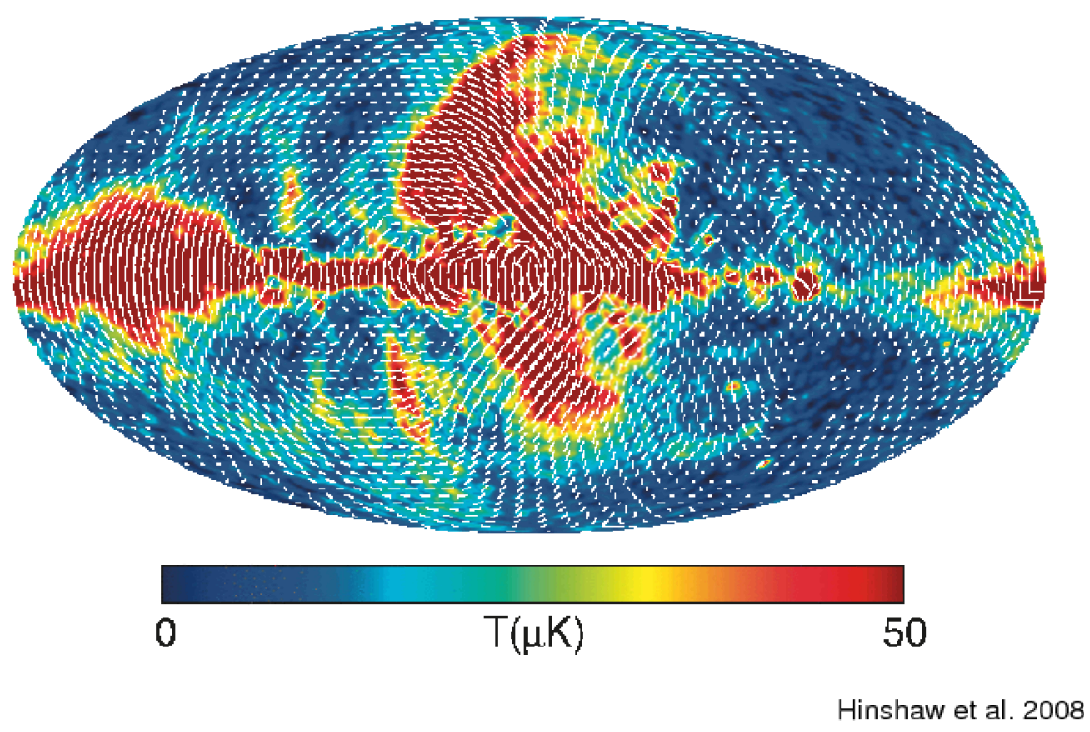

Figure 1: The WMAP measurements of polarization at $23 \mathrm{GHz}$ map the large-scale distribution of polarized synchrotron emission (Mollweide projection in Galactic coordinates). White lines indicate the polarization angle $\gamma_{d}$ and amplitude, and are oriented perpendicular to the magnetic field.

for assessing the validity of the chosen model.

\subsection{Synchrotron emission}

Synchrotron emission results from the acceleration of cosmic-ray electrons in the magnetic field of the Galaxy. For a power-law distribution of electron energies, $N(E) \propto E^{-p}$, propagating in a uniform magnetic field, the resulting emission is partially polarized with fractional linear polarization

$$
f_{s}=\frac{p+1}{p+7 / 3}
$$

aligned perpendicular to the magnetic field (Rybicki \& Lightman 1979). The frequency dependence of synchrotron emission is also related to the electron energy distribution, $T(\nu) \propto \nu^{\beta}$, with spectral index

$$
\beta=-\frac{p+3}{2}
$$

where $T$ is in units of antenna temperature. For spectral index $\beta \approx-3$ observed at microwave frequencies, synchrotron emission could have fractional polarization as high as $f_{s} \sim 0.75$, although in practice this is almost never observed. Line-of-sight and beam averaging effects tend to reduce the observed polarization by averaging over regions with different electron energy distribution or magnetic field orientation. At frequencies below a few GHz, Faraday rotation will induce additional depolarization. 


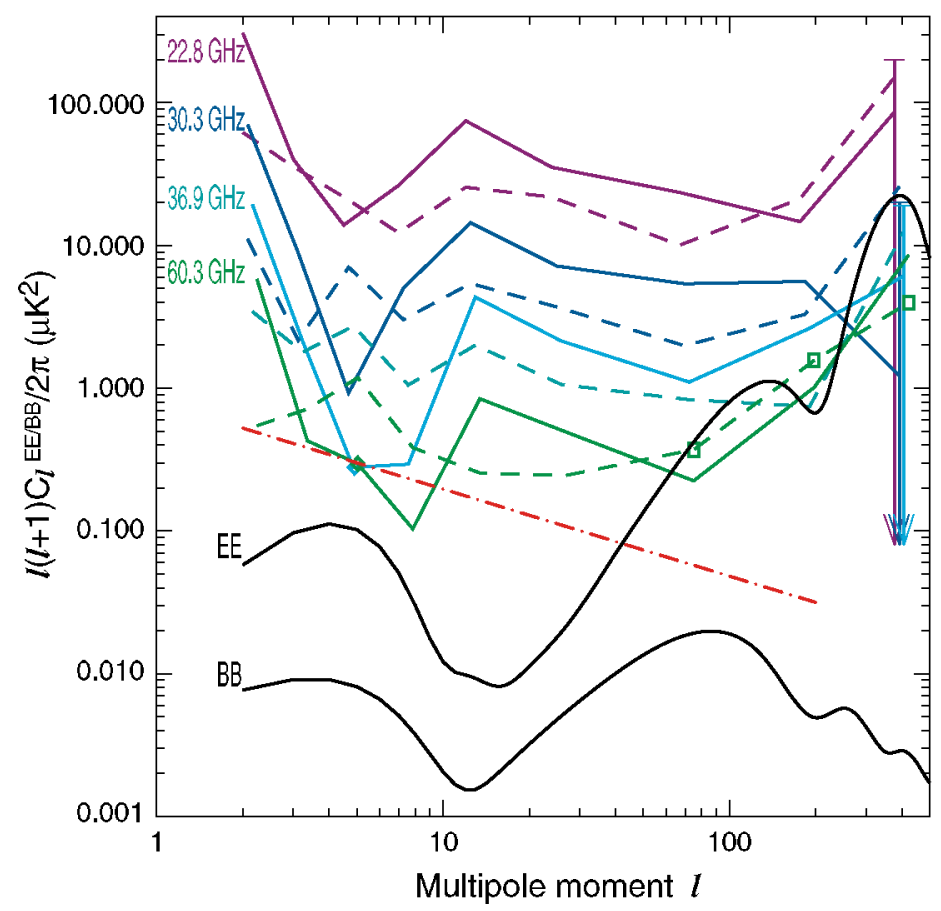

Figure 2: Power spectra for the WMAP 3-year polarization maps are dominated by foreground emission. Solid lines show EE spectra and dashed lines show BB spectra outside the Galactic plane; the color indicates frequency band. The rise in power for $\ell>100$ is an artifact of the instrument noise. The red dot-dashed line shows the estimated BB foreground power at $60 \mathrm{GHz}$, using a parameterized model fit to the multi-frequency data. Diamonds (EE) and boxes (BB) indicate points with negative values. The cosmological model shown has $r=0.3$ and $\tau=0.09$. From Page et al. (2007).

The polarized synchrotron emission may be parameterized as

$$
\begin{aligned}
& Q(\hat{n})=T_{s}^{P(\hat{n}) \cos (2 \gamma(\hat{n}))}\left(\frac{\nu}{\nu_{0}}\right)^{\beta_{s}(\hat{n})+C(\hat{n}) \log \left(\nu / \nu_{0}\right)} \\
& U(\hat{n})=T_{s}^{P}(\hat{n}) \sin (2 \gamma(\hat{n}))\left(\frac{\nu}{\nu_{0}}\right)^{\beta_{s}(\hat{n})+C(\hat{n}) \log \left(\nu / \nu_{0}\right)}
\end{aligned}
$$

where $Q(\hat{n})$ and $U(\hat{n})$ are the Stokes parameters in direction $\hat{n}, T_{s}^{P}(\hat{n})$ is the (polarized) amplitude $T^{P}=\sqrt{Q^{2}+U^{2}}, \gamma(\hat{n})$ is the polarization angle, $\beta_{s}(\hat{n})$ is the spectral index, and $C(\hat{n})$ parameterizes any spectral curvature (flattening or steepening) relative to a pure power law.

The Wilkinson Microwave Anisotropy Probe (WMAP) provides our best estimate of the synchrotron morphology on angular scales of a few degrees or larger. Figure 1 shows the full-sky WMAP observations at $23 \mathrm{GHz}$ (Page et al. 2007; Hinshaw et al. 2008). At $4^{\circ}$ pixelization, the signal to noise ratio for polarized synchrotron emission is better than three over $90 \%$ of the sky. The polarization angle has coherent structure over large swaths of the sky, creating significant emission at low multipole moments $\ell$. Figure 2 shows the power spectra of the WMAP polarization data from 23 to $94 \mathrm{GHz}$ (Page et al. 2007). The power spectra are dominated by foreground emission with roughly equal power in E- and B-modes, which, averaged over the high-latitude sky, are brighter than the CMB polarization even at the minimum near 70-100 GHz. A simple parameterization of the foreground emission in Page et al. (2007) gives a power spectrum with $\ell(\ell+1) C_{\ell} / 2 \pi \propto \ell^{-0.6}$, 

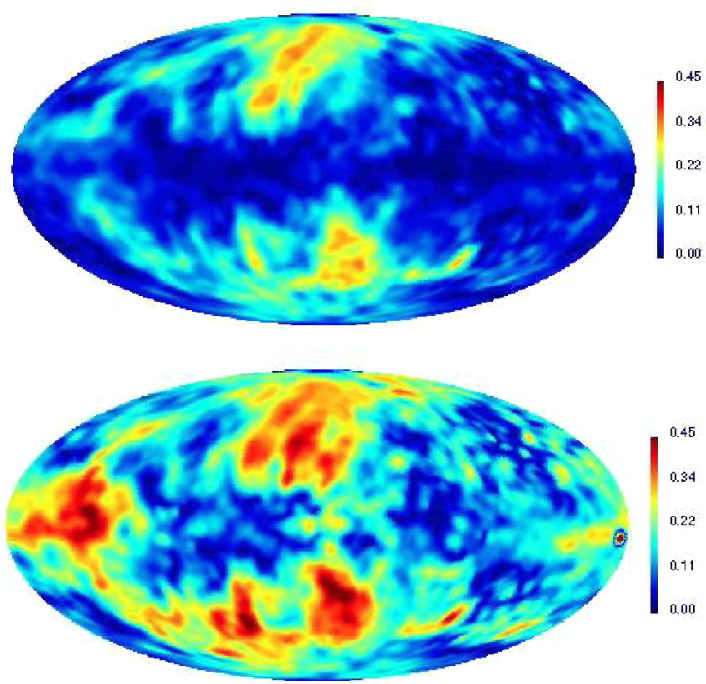

Figure 3: Estimated polarization fraction $f=P / I$ at $5^{\circ}$ resolution. The polarization $P=\sqrt{Q^{2}+U^{2}}$ is well estimated from the WMAP K band data (Page et al. 2007) and the intensity $I$, which is less certain, is modeled excluding (top), and including (bottom), an unpolarized 'anomalous' dust component (from Miville-Deschenes et al. (2008)).

shown in red in Figure 2. This is consistent with the power spectrum for synchrotron emission observed from radio maps (see e.g. La Porta et al. (2008)).

We can estimate the fractional polarization of synchrotron emission by comparing the WMAP $23 \mathrm{GHz}$ map to an estimate of the unpolarized synchrotron emission. The synchrotron intensity suffers from confusion between synchrotron, free-free, and other microwave components, so extracting this signal from observed sky maps depends on modeling assumptions. Kogut et al. (2007) use the WMAP three-year maximum-entropy map of synchrotron emission as a tracer of the intensity (Hinshaw et al. 2007), finding the Galactic plane region $\left(|b|<5^{\circ}\right)$ to be largely depolarized, with mean fractional polarization $f_{s}=P / I=0.05$. The North Galactic spur region at mid-latitudes has fractional polarization $f_{s} \approx 0.3$ while the high latitude sky outside the P06 mask has a very broad distribution with mean $f_{s}=0.15$ (Kogut et al. 2007). The synchrotron polarization is consistent with the magnetic field having rough equipartition between a large-scale smooth component and a smaller scale turbulent component (Page et al. 2007). Miville-Deschenes et al. (2008) estimate the polarization fraction at $23 \mathrm{GHz}$ for a set of synchrotron intensity maps. One map is derived from $W M A P$ data assuming that no anomalous dust emission contributes at $23 \mathrm{GHz}$, and gives a polarization fraction, shown in Figure 3, similar to the one studied in Kogut et al. (2007). An alternative intensity map is derived from WMAP data allowing for an anomalous unpolarized emission component to contribute to the total observed intensity. This results in a modified estimate for the synchrotron intensity, and an increase in polarization fraction over much of the sky, also shown in Figure 3. In this second map, there is a larger polarization fraction in the anti-center region compared to the inner galaxy. These estimates for the polarization fraction would be modified if the anomalous component(s) had a non-negligible polarization fraction. We note however that despite these uncertainties in the polarization fraction, our estimate of the large-scale polarization of synchrotron emission, $P=\sqrt{Q^{2}+U^{2}}$, is robust at $23 \mathrm{GHz}$.

The WMAP data provide a reasonable signal to noise ratio for the polarized synchrotron morphology on angular scales of a few degrees or larger. The spectral index $\beta_{s}(\hat{n})$ is less well constrained. 
Estimates of the spectral index for unpolarized synchrotron emission suffer from confusion with other emission components, while estimates based on polarization data (where confusion is less severe) suffer from the weaker synchrotron signal at frequencies above $23 \mathrm{GHz}$. Figure 4 shows the observed polarization variance $\left\langle P^{2}\right\rangle$ of the WMAP data as a function of frequency for $4^{\circ}$ pixelization. The spectra are consistent with a superposition of two power-law components, with synchrotron $\beta_{s} \approx-3$ and dust $\beta_{d} \approx 2$. The CMB contributes lower power relative to the foreground components. Kogut et al. (2007) find that the synchrotron spectral index steepens off the plane by a modest amount $\Delta \beta_{s} \sim 0.2$. Gold et al. (2008) map the synchrotron spectral index using a combined analysis of the temperature and polarization data, finding a trend towards a steepening of the spectral index off the Galactic plane. A single index of $\beta_{s}=-3.3$ currently works sufficiently well outside the Galactic plane for cleaning the WMAP maps (Page et al. 2007).

Similar problems affect estimates of the synchrotron spectral curvature. The spectral index depends on the energy spectrum of cosmic ray electrons (Eqn. 2). Higher energy electrons lose energy more rapidly, which can lead to a steepening or break in the synchrotron spectrum. The frequency of the break is not well known, but is thought to vary with position on the sky and be in the range 10-100 GHz, corresponding to a break in the electron spectrum at the energy where the characteristic cooling time and the escape time are the same. To a large extent, efforts to characterize any spectral steepening are limited by confusion with thermal dust emission. The effects of steepening for the synchrotron component are largest near the foreground minimum near $70-100 \mathrm{GHz}$, where the amplitudes of synchrotron and thermal dust emission are comparable. New measurements of polarized dust emission at frequencies above $100 \mathrm{GHz}$, from the Planck satellite and other experiments, will help break this degeneracy and provide tighter limits on both the dust and synchrotron spectra. Low frequency observations from experiments at 5-15 GHz, including the C-Band All Sky Survey (C-BASS), will also help determine the frequency dependence of the polarized synchrotron emission.

Little information is available for synchrotron polarization on angular scales below $1^{\circ}$. Existing measurements on small scales are heavily affected by Faraday rotation at low frequencies. Figure 5 shows a polarization map with angular resolution $36^{\prime}$ from the Dominion Radio Astrophysical Observatory at $1.4 \mathrm{GHz}$ (Wolleben et al. 2006). Although some features such as the North Galactic spur at high latitude and the Fan region near longitude $150^{\circ}$ are recognizable from the polarization map at $23 \mathrm{GHz}$ (Fig 1), the $1.4 \mathrm{GHz}$ map is dominated by Faraday depolarization, whereas the 23 $\mathrm{GHz}$ emission has almost no Faraday rotation. Similar depolarization affects interferometric maps at finer angular resolution (Uyaniker et al. 2003; Taylor et al. 2003), limiting their utility for foreground modeling at millimeter wavelengths.

An additional component of synchrotron emission that may be constrained with future polarization data is anomalously hard synchrotron emission towards the Galactic center. This is known as the WMAP 'Haze', found by Finkbeiner (2004); Dobler \& Finkbeiner (2008a); Bottino et al. (2008) in analyses of the WMAP data. This does not have a conclusively confirmed origin, but as it is confined to the Galactic center, it is discussed in more detail in the companion CMBPol document on foreground science (Fraisse et al. 2008).

\subsubsection{Modeling considerations}

Given the spatial variation in the observed synchrotron polarization fraction, it is unrealistic to model the polarized signal as a synchrotron intensity scaled by a single global polarization fraction, as illustrated in Figure 3. This implies that for a given frequency, the emission in every pixel should be modeled with at least two parameters for the synchrotron $\mathrm{Q}$ and $\mathbf{U}$ (or P and $\gamma$ ). In terms of the spectral behavior, the power law approximation seemed to be sufficient for cosmological analyses well outside the plane for WMAP (Page et al. 2007; Gold et al. 2008). However, a power 


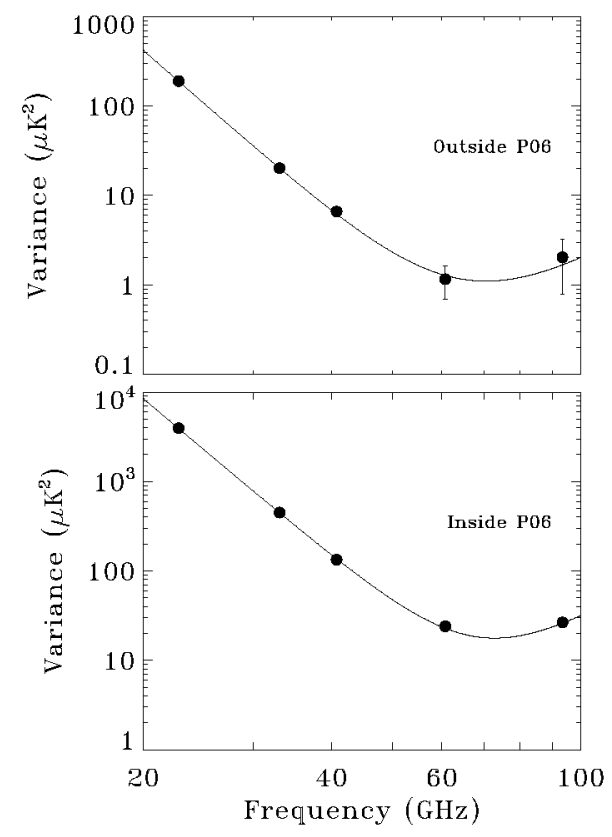

Figure 4: Spectrum of the variance in the WMAP polarization data, computed at each frequency from the cross-spectra of one-year maps, to avoid a bias from instrument noise. The spectra are consistent with power-law synchrotron and dust emission, with synchrotron $\beta_{s} \approx-3$. The P06 cut excludes $25 \%$ of the sky in the Galactic plane. From Kogut et al. $(2007)$.

law fit in smaller pixels, and a curvature fit either globally or in pixels, are likely necessary when pushing the foregrounds down another factor of 10-100. Values for the synchrotron index in the range $-3.5 \lesssim \beta_{s} \lesssim-2.5$ are physically reasonable, with index curvature expected to lie in the range $-0.5 \lesssim C \lesssim 0.5$, consistent with current observations. Additional parameters beyond the curvature may be required to quantify the frequency dependence, although they are not necessary to fit current data. Diffusion of electrons limits the possible spatial variation of the spectral index at small angular scales (see e.g., Strong et al. (2007)), so models where the index is fit in larger regions may be considered. In principle the spectral indices of the observed synchrotron intensity and polarization should be modeled with independent parameters, due to possible depolarization effects from the Galactic magnetic field, although a difference between the two has not yet been conclusively detected. This is due to both uncertainty in the spectral index of the synchrotron intensity, and the instrumental noise levels of the polarization measurements.

\subsection{Dust emission}

Galactic emission in the $100-6000 \mathrm{GHz}$ frequency range $(\lambda=50-3000 \mu \mathrm{m})$ is dominated by thermal emission from warm $(\sim 10-100 \mathrm{~K})$ interstellar dust grains. Here 'thermal' refers to emission from thermal fluctuations in the electric dipole moments of grains. The spectral shape of this emission is generally modeled with one or more thermal components modified by a frequency-dependent emission. That is, for a single temperature, the intensity is proportional to $I(\nu) \sim \nu^{\beta} B_{\nu}(T)$, where $B_{\nu}(T)$ is the Planck function at frequency $\nu$ and temperature $T$. Multiple temperatures and spectral indices $(\beta)$ are often needed to model the intensity spectrum at any single point on the sky. Using 


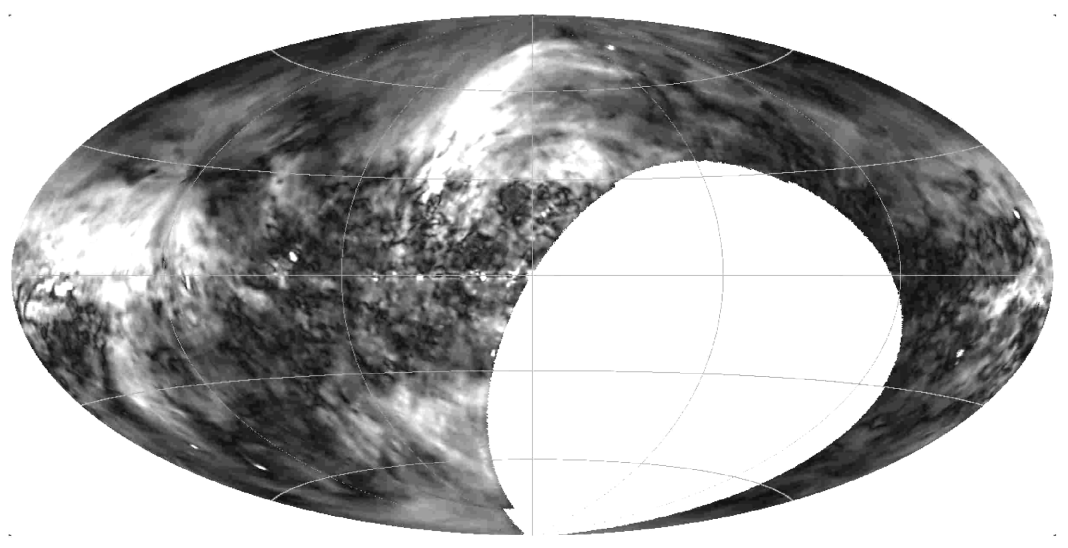

Figure 5: Polarization intensity measured at 1.4 GHz. The prominent Faraday depolarization limits use of low-frequency maps for foreground modeling, despite the finer angular resolution. From Wolleben et al. (2006). The gray-scale runs from 0 to $450 \mathrm{mK}$ in brightness temperature.

data from the all-sky surveys of IRAS at $100 \mu \mathrm{m}$ (Neugebauer et al. 1984), and COBE/DIRBE at 100 and $240 \mu \mathrm{m}$ (Fixsen et al. 1998), Finkbeiner et al. (1999) have modeled this Galactic dust emission using two temperature components, with $\left\langle T_{1,2}\right\rangle=9.5$ and $16 \mathrm{~K} ; \beta_{1,2}=1.7$ and 2.7. Both components are in equilibrium with the interstellar radiation field. They then used this model to predict thermal dust emission at microwave frequencies. The resulting model is calibrated from $C O B E / D I R B E$ and has the angular resolution of IRAS $\left(\approx 6^{\prime}\right)$. The predicted signal, shown in Figure 6 , has been found consistent with WMAP total intensity data (Bennett et al. 2003), especially in the 61 and $94 \mathrm{GHz}$ ( $\mathrm{V}$ and $\mathrm{W}$ ) bands where the dust contribution is greatest. This model has become the standard template for removing the contribution of Galactic dust from microwave observations.

To date there are no similar models that can be used to remove the polarized emission from these same interstellar dust grains. WMAP polarization observations (Page et al. 2007; Kogut et al. 2007; Dunkley et al. 2008b) at $\mathrm{V}$ and $\mathrm{W}$-band show evidence of a dust polarization fraction ranging from $\sim 1 \%$ in the direction of the Galactic center to a few percent at intermediate latitudes, consistent with the observations of Archeops at $353 \mathrm{GHz}$ (Ponthieu et al. 2005). However, due to the high noise level of the $\mathrm{V}$ and $\mathrm{W}$-band polarization measurements, an estimate of the polarization angle is possible only on very large angular scales $\left(\gtrsim 4^{\circ}, \ell \sim 50\right)$, and does not have high signal-to-noise. Therefore, additional information is needed in order to remove polarized dust foregrounds on both large and smaller angular scales. However, this is not a limitation to initiating a CMBPol mission, as a dust model can be generated from the data if the mission has a rich enough data set in terms of frequency coverage and number of channels. This scenario occurred for both IRAS and WMAP, where data was used both to construct foreground templates and to measure the CMB spectrum.

\subsubsection{Dust Alignment}

Thermal dust polarization results from aspherical dust grains that have been aligned by interstellar magnetic fields. The exact grain alignment mechanism is complex so we provide only a brief description here. Basic alignment requires the grain's small axis, with the largest moment of inertia, to align with the spin axis, followed by alignment of the the spin axis with the local magnetic field (e.g., Davis \& Greenstein 1951; Whittet 2003; Lazarian 2003, 2007; Roberge 2004). While other alignment mechanisms (e.g., mechanical alignment; Gold 1952) may dominate in some select environments, the above mechanism is favored in conditions prevalent throughout most of the ISM. 


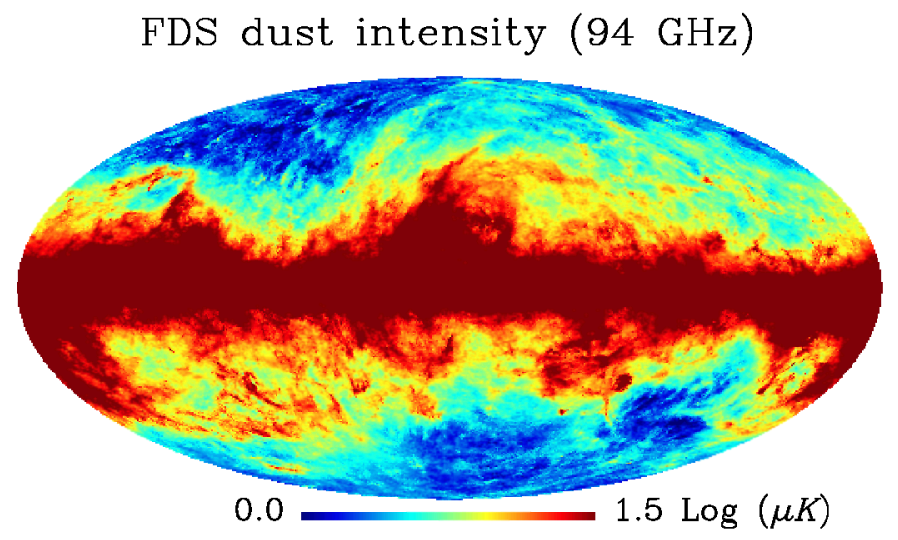

Figure 6: Thermal dust intensity observed by IRAS and extrapolated to $94 \mathrm{GHz}$ by Finkbeiner et al. (1999). This map is commonly used to identify regions of expected low dust emission, with the minimum at Galactic coordinates $(l, b) \sim(240,-70)$. Although the dust polarization fraction is expected to vary spatially, this map is also useful for identifying regions of potential low dust polarization.

Modern grain alignment theory favors grain-photon interactions, or radiative torques, as the mechanism by which grains achieve high-angular velocities and subsequent alignment (e.g., Dolginov 1972; Dolginov \& Mytrophanov 1976; Draine \& Weingartner 1996, 1997; Lazarian \& Hoang 2007; Hoang \& Lazarian 2008). The result of these mechanisms is to align the longest grain axis perpendicular to the local magnetic field. All grains will emit, and absorb, most efficiently along the long grain axis. Therefore, polarization is oberved perpendicular to the aligning field in emission, but parallel to the field in absorption or extinction.

Polarized thermal emission at $\lambda=60-1000 \mu \mathrm{m}$ has been observed in a wide variety of dusty Galactic objects and typically has a polarization fraction of $0.5-10 \%$ (e.g., Dotson et al. 2000; Curran \& Chrysostomou 2007; Matthews et al. 2008; Dotson et al. 2009). To date, polarization from the extinction of background starlight at near-visible wavelengths, believed to be due to differential absorption, has been observed for more than 10,000 stars, typically with values $\sim 1-4 \%$ (e.g., Heiles 2000; Berdyugin et al. 2004). Both types of observations have been used to study magnetic field structure throughout the Galaxy (e.g., Chuss et al. 2003; Schleuning 1998; Matthews et al. 2001; Fosalba et al. 2002; Zweibel \& Heiles 1997; Pereyra \& Magalhães 2004, 2007) as well as to investigate the alignment mechanism and physical dust characteristics (e.g., Hildebrand \& Dragovan 1995; Aitken 1996; Hildebrand et al. 1999; Whittet et al. 2001). For further discussion on grain alignment see Fraisse et al. (2008).

\subsubsection{Polarized Dust Emission}

Current instrument sensitivity limits preclude a comprehensive characterization of the properties of polarized dust emission at millimeter wavelengths. Existing measurements of diffuse regions are limited to large beams (intrinsic or binned) usually greater than $\sim 2^{\circ}$ (e.g., Ponthieu et al. 2005; Page et al. 2007; Takahashi et al. 2008). High-sensitivity measurements of polarized dust have thus far been limited to dense regions of the ISM. These are generally regions in the Galactic plane (with optical extinctions exceeding $A_{V} \sim 20$ magnitudes) which are avoided for CMB analysis. Because dense dusty regions involve multiple populations of dust grains in a complex magnetic field structure, simple parameterizations describing dust behavior are likely to be significantly different 
in dense Galactic regions than in diffuse regions targeted for CMB observations, so care must be taken when using these measurements to predict emission characteristics in diffuse regions. However, useful information can still be gleaned.

Multi-wavelength observations of dense clouds in the Galactic plane at far-infrared through millimeter wavelengths show clear evidence of a wavelength-dependent polarization in terms of both the fractional polarization (Hildebrand et al. 1999; Vaillancourt 2002, 2007) and position angle (e.g., Schleuning et al. 2000). In a sample of the most massive clouds, the spectrum has a minimum at approximately $350 \mu \mathrm{m}(860 \mathrm{GHz})$ and can vary by up to a factor of 4 in the $60 \mu \mathrm{m}-1 \mathrm{~mm}$ wavelength range. These changes in polarization amplitude and position angle are attributed to multiple populations of dust grains with different temperatures, emissivities, and polarization efficiencies, as well as differences in the aligning magnetic field direction. Draine \& Fraisse (2008) demonstrate the expected variation of the polarization fraction with wavelength due to a mixture of silicates and carbonaceous grains.

The structure in the $50-1000 \mu \mathrm{m}(300-6000 \mathrm{GHz})$ polarization spectrum of dense clouds results from the existence of multiple dust temperature components at $20-60 \mathrm{~K}$ (Vaillancourt 2002). On the other hand, lower frequency emission from the diffuse IR cirrus $(\nu<300 \mathrm{GHz})$ is expected to be dominated by a single cold dust component ( $T \sim 10 \mathrm{~K}$ ), in thermal equilibrium with the interstellar radiation field (Finkbeiner et al. 1999). Therefore, although multiple polarization domains are also likely to exist along most lines of sight in low-extinction regions (preferred for CMB analysis) far outside the Galactic plane, this indicates that the polarization fraction will be nearly frequencyindependent in diffuse clouds at $\lambda>1 \mathrm{~mm}, \nu<300 \mathrm{GHz}$ (Hildebrand \& Kirby 2004).

\subsubsection{Starlight Polarization}

Background-starlight polarization is only possible in regions of fairly low extinction ( $A_{V}$ less than a few magnitudes for near-infrared observations) where near-visible photons have sufficient meanfree-path to traverse the ISM. This makes it a feasible tool for inferring the Galactic magnetic field in regions of interest for CMB analysis. However, the extinction places a practical limit on the most distant stars for which polarization can be observed. The vast majority of observed high-latitude stars are within $1 \mathrm{kpc}$ of the Sun, however at low latitude some luminous stars as far away as 2 kpc contribute to the measured polarization signal (Heiles 2000; Fosalba et al. 2002). Despite this limitation, recent analyses of such measurements suggest that they do contain information about the uniform and random components of the magnetic field on large scales (Fosalba et al. 2002). In the foreground polarization model inferred from WMAP data, Page et al. (2007) and Kogut et al. (2007) showed that the polarization angle derived from starlight polarization provides a slightly better fit to the data than the synchrotron polarization angle. Measurements of polarization for stars of different distances can also reveal the 3-D distribution of magnetic field orientations averaged along the line of sight.

Tables 1 and Figure 7 show an analysis from Fosalba et al. (2002) using the catalog of starlight polarization data from Heiles (2000). This analysis includes only stars with reliable distance and extinction estimates, and small polarization measurement uncertainties ( 5513 stars, $60 \%$ of the Heiles catalog). Low-latitude stars have large polarization fractions and extinctions $\left(f_{d}=P / I \approx 1.7 \%\right.$, $E(B-V) \approx 0.5 \mathrm{mag})$, while high-latitude sources exhibit significantly lower values $\left(f_{d} \approx 0.5 \%\right.$, $E(B-V) \approx 0.15 \mathrm{mag})$. As shown in Figure 7 there is a strong net alignment of starlight polarization vectors with the Galactic plane (see lower panel) as well as a clear alignment with the spherical shell of Loop 1 as seen from the polarization vectors in local clouds (upper panel). The amplitude of the polarization vectors are also found to vary nearly sinusoidally with Galactic longitude (Fosalba et al. 2002). The plane-of-sky polarizations are near-minimum towards lines-of-sight tangent to Galactic spiral arms and near-maximum towards lines-of-sight perpendicular to spiral arms. This observation 


\begin{tabular}{ccccc}
\hline Latitude & Distance & No. Stars $(\%)$ & $f=P / I(\%)$ & $\mathrm{E}(\mathrm{B}-\mathrm{V})$ \\
\hline & Total & $4114(75)$ & 1.69 & 0.49 \\
Low Latitude & Nearby & $1451(26)$ & 0.94 & 0.29 \\
$\left(|b|<10^{\circ}\right)$ & Distant & $2663(48)$ & 2.09 & 0.60 \\
\hline & Total & $1399(25)$ & 0.45 & 0.15 \\
High Latitude & Nearby & $1315(24)$ & 0.42 & 0.14 \\
$\left(|b|>10^{\circ}\right)$ & Distant & $84(1)$ & 0.89 & 0.26 \\
\hline
\end{tabular}

Table 1: Mean stellar parameters by distance and Galactic latitude (b) from Fosalba et al. (2002). 'Nearby' and 'distant' denote stars within and beyond $1 \mathrm{kpc}$, respectively. The quantities in parentheses denote the fraction, in percent, of all stars in the sample.

is consistent with a model in which the Galactic magnetic field follows the spiral arm structure (e.g. Heiles 1996; Beck 2007). Further reconstruction of the three-dimensional Galactic magnetic field structure requires complementary observations including radio synchrotron polarization, IRsubmillimeter dust polarization and Faraday rotational measures from distant pulsars and quasars (e.g., Han et al. 2006; Han \& Zhang 2007; Han 2007). New stellar polarization data from an optical survey described in Magalhães et al. (2005) is expected in the next few years, and proposals for new observations have been submitted. See Fraisse et al. (2008) for further discussion.

\subsubsection{Modeling considerations}

The expectation of changing polarization with position indicates that at a given frequency, two parameters are required to quantify the $\mathrm{Q}$ and $\mathrm{U}$ Stokes parameters (or $\mathrm{P}, \gamma$ ) in each pixel. The fractional polarization $f=P / I$ is expected to be everywhere less than $\sim 15 \%$ (Draine \& Fraisse 2008 ), but may range from less than $1 \%$ in some regions to $10-15 \%$ in highly polarized regions. The dust intensity can be used to provide additional constraints and will be better understood with Planck observations in hand. Possible models for the polarization fraction include inverse variation with dust column density, or with some measure of the interstellar radiation field intensity. The number of parameters required to model the frequency dependence of the polarized intensity in a given direction is not yet established, although Sec 2.2 .2 indicates that optimistically one may be sufficient at frequencies below $300 \mathrm{GHz}$, as the dust polarization is likely to be dominated by a single component. A simple power law index can be modeled with one parameter in each pixel, and could take values in the range $1 \lesssim \beta_{d} \lesssim 3$, with $\beta_{d}=1.7$ fitting current temperature and polarization data at $\nu<100 \mathrm{GHz}$. However, since the dust consists of at least two components with different indices (Finkbeiner et al. 1999), additional parameters may be required to quantify their frequency dependence. Extrapolating from frequencies above $\sim 300 \mathrm{GHz}$ is likely to lead to modeling errors, and therefore bias the estimated signal if power law behavior is assumed.

\subsection{Free-free emission}

Free-free (bremsstrahlung) emission is due to electron-electron scattering from warm $\left(T_{e} \approx 10^{4} \mathrm{~K}\right)$ ionized gas in the ISM. In the optically thin regime, and at radio frequencies, the brightness temperature is given by $T_{b}=8.235 \times 10^{-2} a T_{e}^{-0.35} \nu_{\mathrm{GHz}}^{-2.1}(1+0.08)(\mathrm{EM})_{\mathrm{cm}^{-6} \mathrm{pc}}$ where $T_{e}$ is the electron temperature, EM is the Emission Measure and $a$ is a factor close to unity (Rybicki \& Lightman 1979; Dickinson et al. 2003). The electron temperature in the warm ionized medium ranges from $\sim 3000$ to $20000 \mathrm{~K}$ but is $\sim 8000 \mathrm{~K}$ at the Solar Galactocentric distance. The spectrum follows a 


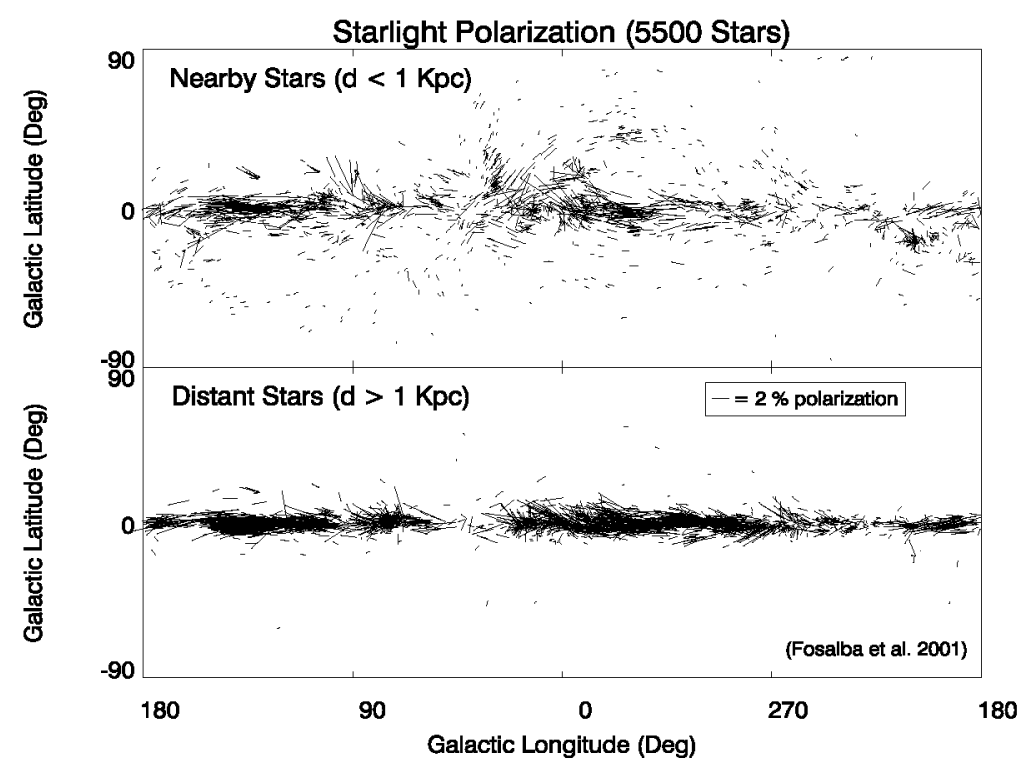

Figure 7: Starlight polarization vectors in Galactic coordinates for a sample of 5513 stars. The upper panel shows polarization vectors in local clouds, while the lower panel displays polarization averaged over many clouds in the Galactic plane. The length of the vectors is proportional to the polarization degree and the scale used is shown in the lower panel. For starlight the direction of polarization is parallel to the magnetic field. From Fosalba et al. (2002).

well-defined power-law with a spectral index of -2.1 showing only a small variation with temperature. At WMAP frequencies, the effective free-free spectral index is $\sim-2.14$ for $T_{e} \sim 8000 \mathrm{~K}$. For modeling purposes, a reasonable prediction for the intensity signal outside the Galactic plane is the H $\alpha$ map compiled by Finkbeiner (2003) with a correction for dust extinction (Bennett et al. 2003), or the MEM map obtained from the WMAP analysis (Gold et al. 2008).

Free-free emission is intrinsically unpolarized because the scattering directions are random. However, a secondary polarization signature can occur at the edges of bright free-free features (i.e. HII regions) from Thomson scattering (Rybicki \& Lightman 1979; Keating et al. 1998). This could cause significant polarization $(\sim 10 \%)$ in the Galactic plane, particularly when observing at high angular resolution. However, at high Galactic latitudes, and with a relatively low resolution, we expect the residual polarization to be $<1 \%$. Therefore, free-free emission is unlikely to be a major foreground contaminant for CMB polarization measurements in clean regions of sky.

\subsection{Additional polarized components}

There are at least two additional dust emission mechanisms that have been suggested in the literature that could produce a low level of polarized emission. A number of high Galactic latitude studies (Kogut et al. 1996a; de Oliveira-Costa et al. 1997; Leitch et al. 1997; Hildebrandt et al. 2007), as well as studies of individual Galactic clouds (Finkbeiner et al. 2004; Watson et al. 2005), have observed emission in excess of that expected from the three foreground components discussed above, namely synchrotron, thermal dust, and free-free emission. This emission has been termed anomalous for the simple reason that its provenance is not completely understood at this time. However, it is clear that this anomalous emission is highly correlated with large-scale maps of far infrared emission from thermally emitting dust grains (e.g., Finkbeiner 2004; Davies et al. 2006; Dobler \& Finkbeiner 
2008a; Dobler et al. 2008). Bennett et al. (2003) suggested that this correlation with the WMAP data could be explained by synchrotron emission from dusty star-forming regions. Alternatively, Draine \& Lazarian (1998a,b, 1999) invoked microwave emission from the dust itself to explain the excess. The hypothesis that this emission is dust-correlated synchrotron is disfavored by WMAP polarization maps since they resemble the $408 \mathrm{MHz}$ synchrotron map at $23 \mathrm{GHz}$, whereas the total intensity map at that frequency much more closely resembles the $100 \mu \mathrm{m}$ dust map. Dust-correlated synchrotron would be completely ruled out by upcoming surveys like C-BASS at $5 \mathrm{GHz}$. Such low frequency surveys will be essential for further tests of these hypotheses as synchrotron and microwave dust emission have divergent spectra below the lowest WMAP frequency at $23 \mathrm{GHz}$.

The correlation of anomalous emission and thermal dust emission has led to two hypotheses in which interstellar dust grains themselves are the source of this emission (see review by Lazarian \& Finkbeiner 2003). In the first mechanism, small (radii $\sim 0.001 \mu \mathrm{m}$ ), rapidly rotating grains emit electric dipole radiation at microwave frequencies (Draine \& Lazarian 1998a,b; Ali-Hä̈moud et al. 2008). In the second mechanism, larger (radii $\gtrsim 0.1 \mu \mathrm{m}$ ), thermally vibrating grains undergo fluctuations in their magnetization, thereby emitting magnetic dipole radiation, also at microwave frequencies (Draine \& Lazarian 1999).

The small 'spinning-dust' grains can be stochastically heated to temperatures approaching $100 \mathrm{~K}$ (Draine \& Li 2001; Ali-Haïmoud et al. 2008) resulting in significant mid-infrared emission $(\lambda \sim 10$ $-30 \mu \mathrm{m}$ ) whereas the larger 'magnetic-dust' grains typically emit in thermal equilibrium in the farinfrared $(\lambda \sim 50-200 \mu \mathrm{m})$. Observations which show stronger correlations between the excess emission and the shorter-wavelength (12 and $25 \mu \mathrm{m}$ ) IRAS bands, as compared to the longer-wavelength bands $(60$ and $100 \mu \mathrm{m}$ ), clearly appear to favor spinning-dust over magnetic-dust (de Oliveira-Costa et al. 2002; Casassus et al. 2006). Furthermore, correlations of the microwave excess with $\mathrm{H} \alpha$ emission peaking at $\sim 40 \mathrm{GHz}$ (Dobler \& Finkbeiner 2008b; Dobler et al. 2008) also favor spinning-dust in the warm ionized medium (WIM), as both $\mathrm{H} \alpha$ and WIM spinning dust are expected to be proportional to the density squared of ions. Despite the strong evidence for spinning dust, emission from magnetic-dust must exist at some level, as large grains are known to exist from observed emission in the far infrared, and contain ferromagnetic material which is depleted from the gas-phase (e.g. Savage \& Sembach 1996; Sembach \& Savage 1996). This is especially important for polarization observations as magnetic dust is predicted to be much better aligned than spinning dust.

The small grains responsible for spinning-dust emission are the same grains responsible for starlight extinction at ultraviolet wavelengths (e.g., Mathis, Rumpl, \& Nordsieck 1977). Theoretical models of grain alignment predict spinning-dust polarization could be as high as $7 \%$ at $2 \mathrm{GHz}$, falling to $\lesssim 0.5 \%$ at frequencies above $30 \mathrm{GHz}$ (Lazarian \& Draine 2000 ). Observationally, the relatively low polarization observed at UV wavelengths suggests that these small grains are inefficiently aligned (Whittet 2004; Martin 2007) and will, therefore, produce little polarized emission at any frequency. The larger vibrating magnetic-dust grains are also responsible for the thermal emission seen at farinfrared and submillimeter wavelengths. There is clear evidence that these grains are well aligned since their polarized thermal emission is observed to be as high as $10 \%$ ( $(2.2 .1)$. Theoretical models suggest that magnetic dipole emission from these grains can reach levels as high as $40 \%$ (Draine \& Lazarian 1999). Additionally, the polarization angle is predicted to exhibit $90^{\circ}$ flips as a function of frequency within the $\sim 1-100 \mathrm{GHz}$ range; at high frequencies the position angle is perpendicular to the aligning magnetic field (parallel to the angles of polarized spinning-dust, thermal dust, and synchrotron emission), but parallel to the field at lower frequencies.

Regardless of the exact emission mechanism, the polarization will need to be explored and accounted for in foreground cleaning, especially since the morphology and frequency dependence of the emission is not particularly well known. The $23 \mathrm{GHz}$ WMAP map, as well as targeted observations of specific Galactic clouds (Mason, Robishaw, \& Finkbeiner 2008; Battistelli et al. 2006; Dickinson et al. 2007) suggest that the polarization of the anomalous emission is low, $\lesssim 5 \%$, 
with a measurement of $3.4_{-1.9}^{+1.5 \%}$ by Battistelli et al. (2006). This is consistent with the models of polarized spinning-dust discussed above, but cannot rule out significant contributions from polarized magnetic-dust emission. It is worth noting however that the challenges of addressing these anomalous mechanisms are not insurmountable, and similar challenges have been addressed successfully with previous experiments including FIRS, DMR, FIRAS, and WMAP. There are many ways to approach and remove foregrounds provided that low noise observations are made at a sufficient range of frequency channels at $20-1000 \mathrm{GHz}$. Prior to the launch of a CMBPol mission, ground based surveys will cover the lower frequency range. In addition, complimentary targeted searches of specific Galactic clouds will continue to constrain the polarization properties.

Finally, it is possible that in addition to these dust emission mechanisms, there may also be other exciting discoveries awaiting us that at present are not in our models.

\subsection{Simulated foreground maps and spectra}

By drawing together current observations and theoretical predictions, we generate simulated maps of the polarized Galactic emission with pixels of side 4 and 0.5 degrees $^{1}$. In anticipation of the Planck satellite mission, the 'Planck Sky Model' (PSM) has been developed (Leach et al. 2008), drawing on observations including WMAP, IRAS, and $408 \mathrm{MHz}$ radio data. The Planck Working Group 2 have developed this model and allowed us to use components of it for this work. For initial forecasting purposes we generate a set of foreground maps with synchrotron and dust components, each with power-law spectral indices. This physical behaviour is over-simplified but useful for preliminary tests. The maps with 4 degree pixels are smoothed with a 7 degree Gaussian beam, and those with 0.5 degree pixels with a 1.5 degree beam.

The synchrotron component is formed at each frequency $\nu$ using:

$$
Q_{\text {synch }}(\nu, \hat{n})=\left(\nu / \nu_{s}\right)^{\beta_{s}(\hat{n})} Q_{s}(\hat{n}), \quad U_{\text {synch }}(\nu, \hat{n})=\left(\nu / \nu_{s}\right)^{\beta_{s}(\hat{n})} U_{s}(\hat{n})
$$

For initial studies, the maps for $Q_{s}, U_{s}$ and $\beta_{s}$ are generated from the model of Miville-Deschenes et al. $(2008)^{2}$. They are formed using

$$
Q_{s}(\hat{n})=f_{s} g_{s}(\hat{n}) \cos \left(2 \gamma_{s}(\hat{n})\right) I_{s}(\hat{n}) \quad U_{s}(\hat{n})=f_{s} g_{s}(\hat{n}) \sin \left(2 \gamma_{s}(\hat{n})\right) I_{s}(\hat{n}),
$$

where $I_{s}$ is the predicted synchrotron intensity at $\nu_{s}=33 \mathrm{GHz}$ extrapolated from observations at $408 \mathrm{MHz}$ (Haslam et al. 1981). The geometric suppression factor $g_{s}$, the polarization angles $\gamma_{s}$, and the polarization fraction $f_{s}$ are obtained using a magnetic field model constrained using the WMAP $23 \mathrm{GHz}$ polarization data (Page et al. 2007) and described in Miville-Deschenes et al. (2008). The geometric suppression factor accounts for spatially varying depolarization due to line-of-sight integration through the Galactic magnetic field, resulting in a lower observed polarization fraction than at the point of emission. The factor does not account for beam dilution.

The simulated spectral index map $\beta_{s}$ is obtained by first estimating the synchrotron intensity at $23 \mathrm{GHz}$, using the polarization maps and the same Galactic magnetic field model described above, assuming an emitted polarization fraction of $f_{s}=0.75$. A power law is then fit to the $408 \mathrm{MHz}$ Haslam intensity data and this estimated $23 \mathrm{GHz}$ intensity map. This ignores any spectral break that one might expect in that frequency range, but a scale dependent spectral index can be added to the simulations. The polarization amplitude, angle, and power law spectral index are shown in Figure 8 .

A simple parameterization for the synchrotron emission is

$$
\ell(\ell+1) C_{\ell}^{\mathrm{Synch}} / 2 \pi=A_{s}\left(\nu / \nu_{0}\right)^{2 \beta_{s}}\left(\ell / \ell_{0}\right)^{m_{s}},
$$

\footnotetext{
${ }^{1}$ We use HEALPix $N_{\text {side }}=16$ and 128

${ }^{2}$ As implemented in the PSM v1.6.2.
} 

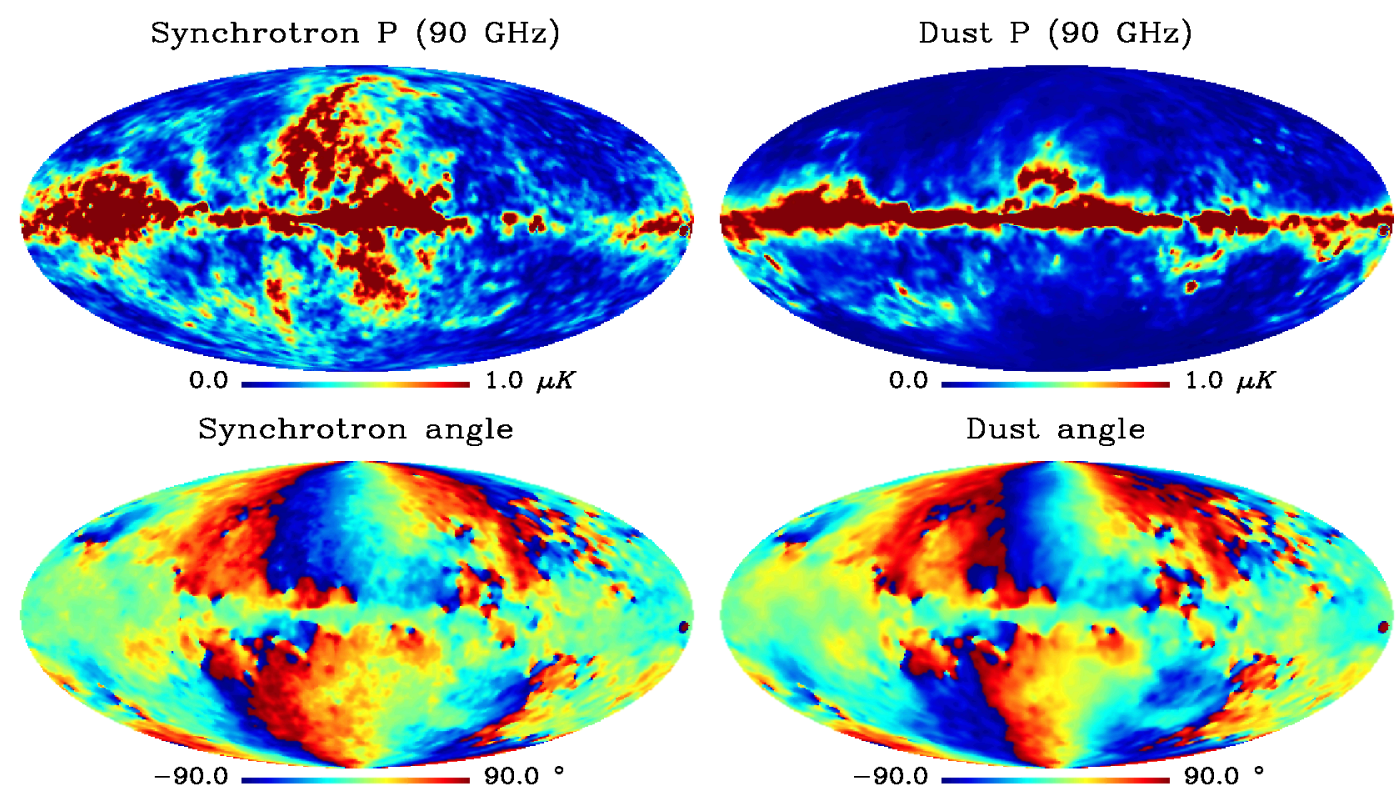

Synchrotron index
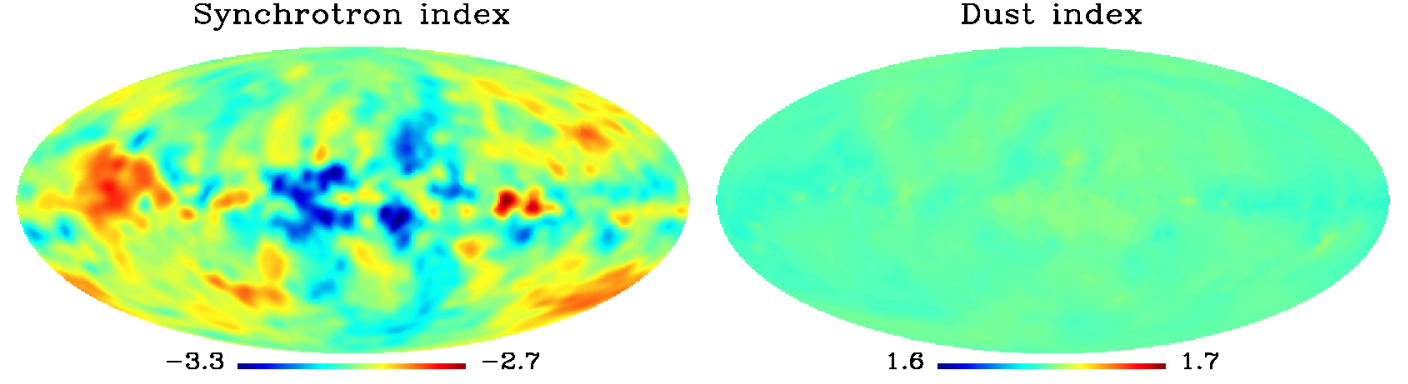

Figure 8: Simulated maps for synchrotron (left) and dust (right) polarization amplitude in antenna temperature, polarization angle, and spectral indices at $90 \mathrm{GHz}$, drawn from the Planck Sky Model and described in the text.

although this oversimplifies the true behavior. Estimates for these quantities are $A_{s} \sim 0.012 \mu \mathrm{K}^{2}$, $\beta_{s} \sim-3.0$, and $m_{s} \sim-0.6$ at $\nu_{s}=90 \mathrm{GHz}$ and $\ell_{0}=10$, based on $W M A P$ observations by Page et al. (2007). Figure 9 shows this spectrum as a function of angular scale, compared to the primordial $\mathrm{CMB}$ signal with tensor-to-scalar ratio $r=0.01$. The estimated synchrotron amplitude (given by the square root of the spectrum) is about three times bigger than the CMB signal for $r=0.01$ at $\ell=100$ over $75 \%$ of the sky at $90 \mathrm{GHz}$, and about ten times bigger than the CMB signal at $\ell=4$. Over the cleanest $3 \%$ of the sky the signal is expected to be comparable to the CMB signal for $r=0.01$.

There is far more uncertainty in the polarization of the thermal dust component, with about an order of magnitude uncertainty in the amplitude, ranging from $\sim 1 \%$ to $10 \%$ polarized. To make simulations, the dust component is formed in a similar way to the synchrotron, using

$$
Q_{\text {dust }}(\nu, \hat{n})=\left(\nu / \nu_{d}\right)^{\beta_{d}(\hat{n})} Q_{d}(\hat{n}), \quad U_{\text {dust }}(\nu, \hat{n})=\left(\nu / \nu_{d}\right)^{\beta_{d}(\hat{n})} U_{d}(\hat{n}) .
$$

The amplitudes and spectral indices are taken from the Planck Sky Model, with the amplitudes formed using

$$
Q_{d}(\hat{n})=f_{d} g_{d}(\hat{n}) \cos \left(2 \gamma_{d}(\hat{n})\right) I_{d}(\hat{n}), \quad U_{d}(\hat{n})=f_{d} g_{d}(\hat{n}) \sin \left(2 \gamma_{d}(\hat{n})\right) I_{d}(\hat{n}) .
$$




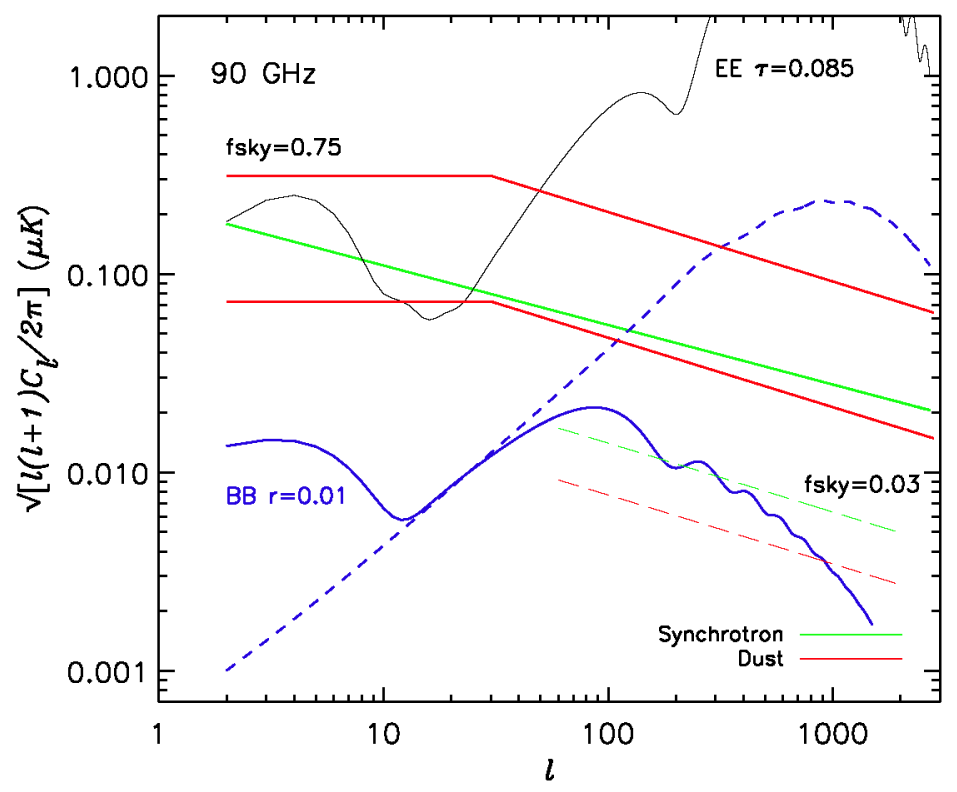

Figure 9: Compilation of measured and predicted E-mode and B-mode amplitudes at 90 $\mathrm{GHz}$ for synchrotron and dust emission compared to the lensed scalar (long dash blue) and tensor (solid blue) CMB signal with $\tau=0.085$ and $r=0.01$. Spectra are shown in antenna temperature units for a sky fraction of $75 \%$, and $3 \%$, in the estimated lowest foreground region. The synchrotron contribution at large scales is constrained by WMAP observations. The dust contribution, and the behavior of both components at $\ell \gtrsim 30$, is uncertain. The red lines show two realistic levels for the predicted dust spectrum, with $5 \%$ and $1.5 \%$ average polarization. Only the lower level is shown for the $3 \%$ sky fraction. Recent simulated dust maps by the Planck collaboration give a B-mode spectrum that lies within these limits, and has a flatter spectrum.

Here $I_{d}$ is the dust intensity at $94 \mathrm{GHz}$ extrapolated from IRAS by Finkbeiner et al. (1999). In the maps we adopt as fiducial estimates, the polarization fraction $f_{d}$ is set to be $5 \%$, and the polarization angles and geometric suppression factor are calculated using the Galactic field model described for the synchrotron simulations. This corresponds to the Planck Sky Model v1.6.2, and assumes that the dust and synchrotron have the same polarization angle. This is not correct along certain lines of sight in the Galaxy (see e.g., Page et al. (2007)), but is used as a first approximation. The resulting map is only 1-2\% polarized due to depolarization effects. In more recent investigations for Planck, a higher polarization fraction of $12 \%$ at emission is assumed, resulting in an observed polarization fraction of $\sim 4 \%$. For the frequency dependence we use power law indices calculated using FDS Model 8 between 84 and $94 \mathrm{GHz}$, with an index $\beta_{d}=1.65$. Figure 8 shows the polarization amplitude, angle, and index for these maps at $\nu_{d}=90 \mathrm{GHz}$. A more realistic model would have multiple polarized dust components which can be modeled by adding their contributions to $Q$ and $U$ given in Eqn. 8 . A two component model is used in the current Planck Sky Model.

Figure 9 shows the angular power spectrum at $90 \mathrm{GHz}$ for the maps outside the P06 sky cut, 
fit with a power law that flattens at large scales. This corresponds to $\sim 1.5 \%$ polarization fraction. The power spectrum currently estimated by the Planck collaboration (PSM v1.6.4), has a higher polarization (3-4\%) and a flatter scale dependence. This model account for more of the turbulent effects in the Galactic magnetic field that tend to flatten the spectrum (Prunet et al. 1998; Prunet \& Lazarian 1999). For comparison we also plot 5\% of the FDS dust intensity. Parameterizing the dust as

$$
\ell(\ell+1) C_{\ell}^{\text {Dust }} / 2 \pi=A_{d}\left(\nu / \nu_{0}\right)^{2 \beta_{d}}\left(\ell / \ell_{0}\right)^{m_{s}}
$$

fails to correctly describe the large scale behavior, but is a useful starting point. Estimates for these quantities over $75 \%$ of the sky fall within typical ranges $1.5<\beta_{d}<2.5,-0.5<m<0.5$, and $A_{d}<0.8 \mu \mathrm{K}^{2}$ for $\ell_{0}=10$ and $\nu_{0}=90 \mathrm{GHz}$. The simulated maps with $1-2 \%$ polarization fraction have $A_{d}=0.004 \mu \mathrm{K}^{2}$ at $\ell=10, \beta_{d}=1.65$, and typically $m=-0.5$. For comparison, at $90 \mathrm{GHz}, 5 \%$ of the FDS intensity corresponds to $A_{d} \sim 0.1 \mu \mathrm{K}^{2}$ at $\ell=10$. An upper limit of $15 \%$ is suggested by Draine \& Fraisse (2008). For a smaller patch of sky, taking a circle of radius 20 degrees in a region of low foreground intensity, the predicted B-mode amplitude is significantly lower, with $A_{d}=6 \times 10^{-5}$ $\mu \mathrm{K}^{2}$, and $A_{s} \sim 0.0002 \mu \mathrm{K}^{2}$ at $\ell=100$ and $\nu=90 \mathrm{GHz}$ for the simulated maps.

Figure 10 shows the total predicted power in the diffuse foregrounds maps as a function of frequency for $\ell=80-120^{3}$. This is based on the PSM model (v1.6.3), with a dust polarization fraction of only $1-2 \%$ percent. The CMB amplitude is for a standard $\Lambda$ CDM model as derived from WMAP five-year results (Dunkley et al. 2008a), including tensor fluctuations with $r=0.01$. Extragalactic sources are not included. The maximum ratio of $\mathrm{CMB}$ to foregrounds occurs at $\sim 100 \mathrm{GHz}$ and moves to slightly higher frequencies for cleaner patches of sky. At $100 \mathrm{GHz}$ and $\ell=100$ the foreground signal is $\sim 5$ times larger than the B-mode signal for $r=0.01$ outside the Galactic plane (in the WMAP Kp2 mask), but in a clean patch of radius 10 degrees the foreground signal is below the CMB signal. However, there is still about an order of magnitude uncertainty in the total polarized dust contribution which should be resolved with observations from Planck, and from ground and balloon-based experiments.

\section{Methods for polarized CMB estimation}

In this section we briefly review and contrast methods commonly used to estimate the polarized CMB signal from raw sky maps. We focus on those that have been applied to current polarization data, and that can be used to forecast the performance of a future mission. For a description of additional methods see e.g., Leach et al. (2008); Delabrouille \& Cardoso (2007).

\subsection{Template cleaning}

A simple foreground cleaning technique assumes that a map of the microwave sky $T(p, \nu)$, at some pixel $p$ and frequency $\nu$, can be described as a superposition of fixed spatial templates $X(p)$ and noise $n(p, \nu)$,

$$
T(p, \nu)=n(p, \nu)+\sum_{i} \alpha_{i}(\nu) X_{i}(p)
$$

\footnotetext{
${ }^{3}$ The power spectra are calculated with PolSpice v2.5.7 (see e.g. Chon et al. (2004)).
} 

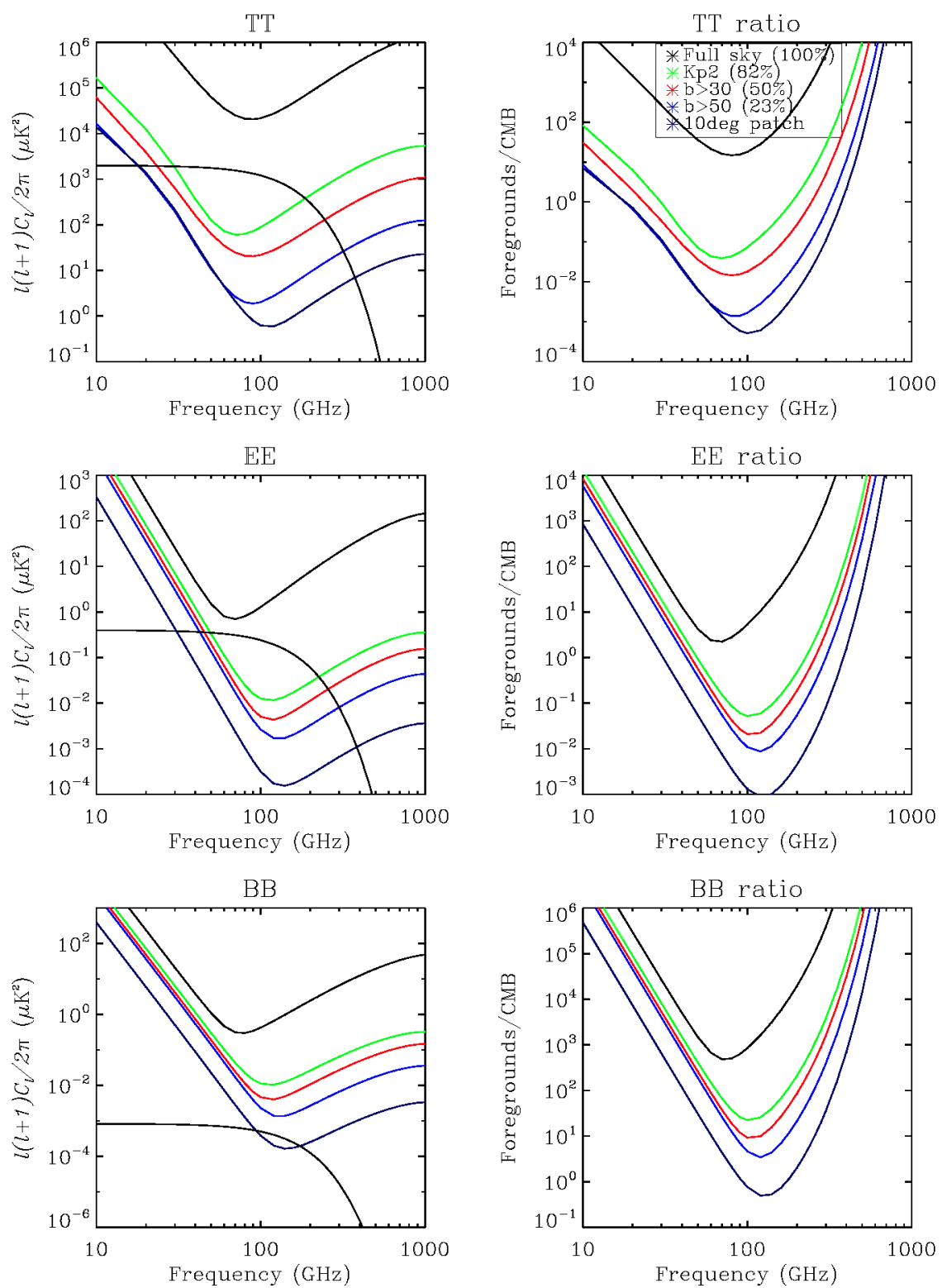

Figure 10: Predicted diffuse foreground power at angular scales $\ell=80-120$, as a function of frequency and sky coverage, compared to a CMB signal with $r=0.01$. From top to bottom are the TT, EE and BB power spectra in antenna temperature. The CMB is constant in thermodynamic temperature and thus decreases with frequency in these units. Left: Total power for different sky coverage: full-sky, $|b|>10^{\circ},|b|>30^{\circ},|b|>50^{\circ}$, and a clean circular patch of radius $10^{\circ}$ centered on $(l, b)=\left(240^{\circ},-70^{\circ}\right)$. Right: Ratio of the total diffuse foreground power to the CMB. The maximum ratio occurs at $\sim 100 \mathrm{GHz}$ and moves to higher frequency for cleaner patches of sky. 

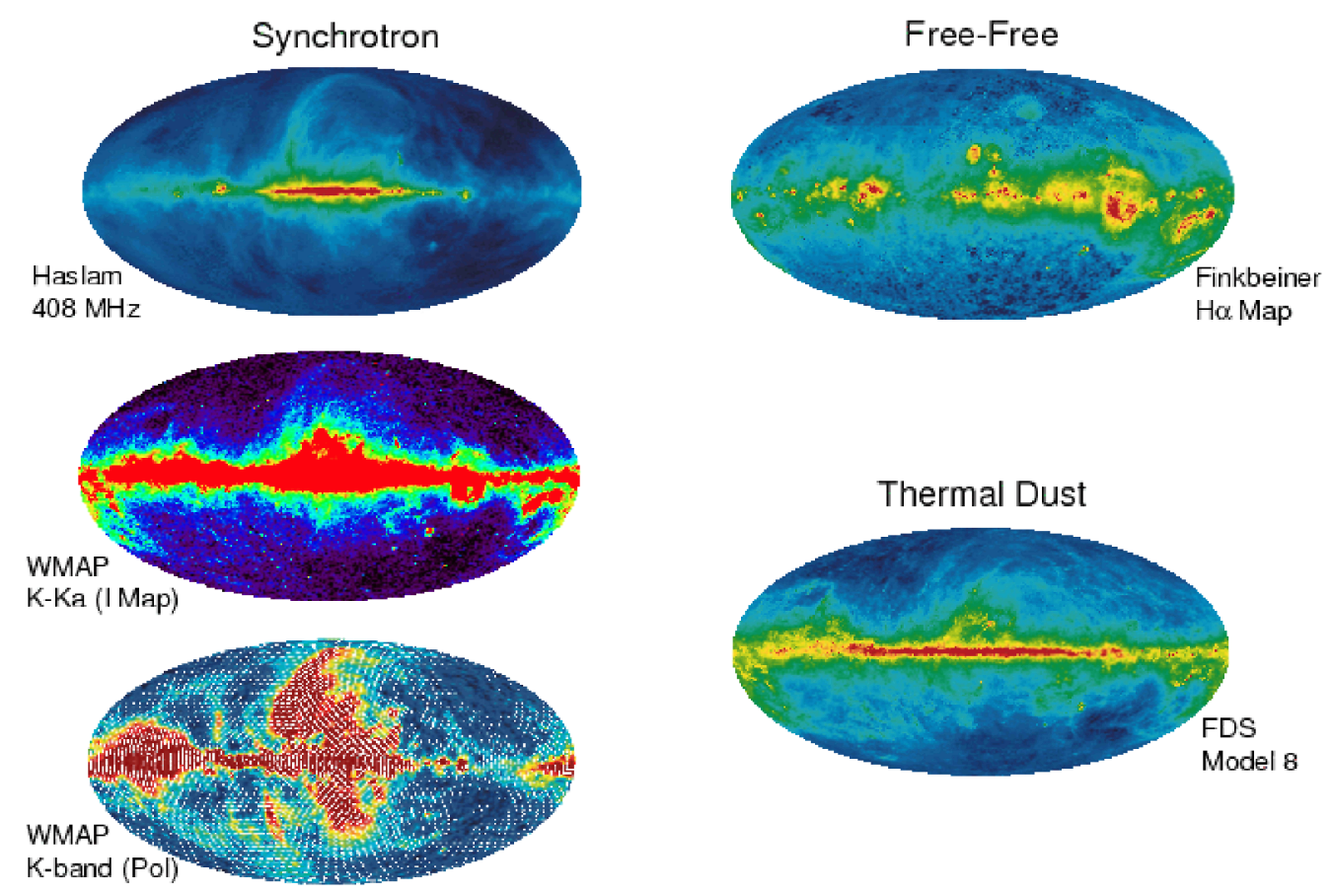

Figure 11: A sample of template maps commonly used to clean polarized and unpolarized maps.

where the coefficients $\alpha_{i}(\nu)$ describe emission traced by the $i^{\text {th }}$ spatial template at observing frequency $\nu$. Provided that the noise covariance matrix (which typically includes both instrument noise and the CMB) is known, Eqn. 10 can be solved for the template coefficients $\alpha_{i}(\nu)$. The noise in the foreground-cleaned map,

$$
T_{c}(p, \nu)=T(p, \nu)-\sum_{i} \alpha_{i}(\nu) X_{i}(p)
$$

is nearly unaffected by template cleaning and retains the properties of the noise in the original map. This is an important consideration for applications requiring accurate propagation of noise into, for instance, estimates of cosmological parameters. The amplitude of the noise in the cleaned map increases as more parameters are fit; however, for most applications there are many more pixels than fitted parameters so the resulting increase is negligible. A typical sky map with $10^{5}$ pixels could simultaneously fit 1000 spatial templates and suffer only a $1 \%$ increase in the noise amplitude after cleaning.

Template cleaning has a number of other attractive features. Since each template is fit to all map pixels simultaneously, the technique makes full use of the spatial information in the template map $X_{i}(p)$. This is important for the non-stationary, highly non-Gaussian emission distribution typical of Galactic foregrounds, allowing cleaning of the entire map even if the foreground emission is faint compared to the noise in each individual pixel. The method also allows multiple template maps to be fit to a single frequency channel, as opposed to pixel-by-pixel techniques which generally require 


\begin{tabular}{|c|ccc|cc|}
\hline Freq & \multicolumn{3}{|c|}{ Unpolarized Coefficient } & \multicolumn{2}{c|}{ Polarized Coefficient } \\
$(\mathrm{GHz})$ & Synchrotron & Free-Free & Dust & Synchrotron & Dust \\
\hline 33 & - & - & - & 0.317 & 0.017 \\
41 & 0.24 & 1.00 & 0.20 & 0.177 & 0.015 \\
61 & 0.06 & 0.65 & 0.47 & 0.060 & 0.037 \\
94 & 0.00 & 0.40 & 1.26 & 0.045 & 0.082 \\
\hline
\end{tabular}

Table 2: WMAP Template Coefficients $\alpha_{i}(\nu)$

at least one frequency channel per foreground component to be fit. In addition, template cleaning is insensitive to spatial correlations between various foreground components. Correlations between the template maps will create non-zero covariance in the fitted parameters $\alpha_{i}$; however, this simply complicates the identification of the associated emission $T_{i}=\alpha_{i} X_{i}$ with the emission mechanism traced by that template, but does not bias the total foreground estimate summed over all templates.

Several cautions apply to template cleaning. The method assumes that the spatial and frequency dependence of each emission component can be described using separable functions for the spatial and frequency dependence, $T(p, \nu)=X(p) f(\nu)$. Many physical emission processes violate this assumption at least to some extent. The spectral index of synchrotron emission, for instance, is known to vary with position on the sky, while dust is known to require at least two temperature components whose ratio also varies across the sky. Over sufficiently large separations in frequency, such spatial variation in the frequency scaling can significantly alter the spatial distribution of the affected emission component. In addition, the technique requires a separate template for each spatially distinct foreground and is thus ineffective as a 'blind' test for foregrounds whose spatial distribution is not well matched to one or more templates. Template cleaning works best when the template map is signal dominated. Noise in the template map will be aliased into the cleaned map and could become significant in clean regions of the sky if the template noise is sufficiently large. While this effect can be estimated using Monte Carlo techniques, some care in template selection and sky cuts should be exercised.

Figure 11 shows a selection of template maps widely used for CMB analyses. Synchrotron emission is often fit using the unpolarized $408 \mathrm{MHz}$ survey (Haslam et al. 1981). Due to concerns over possible spatial variations in the synchrotron spectral index, the WMAP team has also constructed a synchrotron-dominated template using the difference of the two lowest frequency channels (Hinshaw et al. 2007). The CMB anisotropy cancels exactly in such a difference map, leaving a dominant synchrotron signal with smaller contributions from free-free and dust, as well as instrumental noise. Provided the template fit (Eqn. 10) includes separate templates for free-free and dust, the resulting linear combination of templates will remove all three foregrounds. As described in Section 2.3, free-free emission from the warm ionized interstellar medium may be traced using $\mathrm{H} \alpha$ emission from the same ionized gas, subject to non-negligible corrections for optical extinction by interstellar dust (Finkbeiner 2003; Schlegel et al. 1998). Dust emission is commonly traced using a model of millimeter-wave dust emission based on the COBE and IRAS data (Finkbeiner et al. 1999), described in Section 2.2 and shown in Figure 6.

Templates for polarized emission are harder to come by. Free-free emission is largely unpolarized, so the WMAP $23 \mathrm{GHz}$ polarization map can be used as a polarized synchrotron template (Page et al. 2007; Gold et al. 2008). No high signal-to-noise ratio tracer of polarized dust emission currently exists. A polarized dust template may be constructed using the Finkbeiner et al. (1999) unpolarized dust model convolved with optical measurements of the dust polarization angle and a geometric model of the Galactic magnetic field along each line of sight (Page et al. 2007). 
Template cleaning is widely used for $\mathrm{CMB}$ analysis. As an example, Table 2 shows the coefficients fitted to the WMAP 5-year data (Gold et al. 2008). Separate cleaning is performed for the polarized and unpolarized maps. The unpolarized maps are fit over the high-latitude sky using three spatial templates: the synchrotron-dominated K-Ka difference map, the Finkbeiner (2003) H $\alpha$ map corrected for extinction, and the Finkbeiner et al. (1999) model 8 dust map evaluated at $94 \mathrm{GHz}$. Since the "synchrotron" template uses both the K and Ka channels at 23 and $33 \mathrm{GHz}$, the template cleaning is applied only to the 3 high-frequency unpolarized bands. The polarized data, at reduced pixel resolution, are fit using the WMAP $23 \mathrm{GHz}$ map as a synchrotron tracer, plus the Page et al. (2007) template for polarized dust.

Template cleaning is remarkably efficient at compressing complicated Galactic foregrounds into a handful of parameters. Nine parameters describe the unpolarized foreground emission from some seven million input pixels, while 8 parameters describe the polarized emission from nearly 20,000 pixels. This simple cleaning technique produces results in agreement with considerably more complicated pixel-by-pixel methods which include additional astrophysical information.

Template cleaning can also lead to surprising results. Figure 12 shows the template coefficient $\alpha(\nu)$ for a template map of thermal dust emission at $240 \mu \mathrm{m}$ fitted to the COBE/DMR microwave maps at 31, 53, and $90 \mathrm{GHz}$ (Kogut et al. 1996a,b). At sub-mm wavelengths the coefficients follow the expected behavior for thermal dust emission, but show an unexpected increase in emission at $\mathrm{mm}$ wavelengths. This "anomalous emission", correlated with the spatial distribution of thermal dust but with spectral index $\beta \approx-2.2$ (in antenna temperature) instead of the values $1.6<\beta_{d}<2$ expected for thermal dust at mm wavelengths, has since been confirmed in a number of data sets. This is the same emission that is discussed in Section 2.4, and although its physical origin is still unclear, it is efficiently removed by template cleaning.

Template cleaning for CMB analysis to date has operated in two distinct regimes. Unpolarized foregrounds at high latitude are typically fainter than the CMB anisotropy, requiring only a light cleaning of weak foregrounds. Polarized foregrounds are comparable to or brighter than the CMB polarization over most of the sky. Current detections of E-mode polarization, at amplitudes comparable to the foregrounds, thus require modest cleaning of bright foregrounds. A search for primordial B-mode polarization will push template cleaning into a third regime, requiring deep cleaning of bright foregrounds. Detecting a signal with tensor to scalar ratio $r=0.01$ at multipoles $\ell<10$ (angular scales $\theta>20^{\circ}$ ) requires reducing the foreground emission by a factor of roughly 20 .

Happily, recent history suggests that such deep cleaning is feasible and should be possible with a future mission. The cosmic infrared background (CIB) observed at wavelengths $125<\lambda<2000 \mu \mathrm{m}$ results from the integrated infrared emission of galaxies at redshift $z \sim 3$. Galactic emission at these wavelengths is brighter than the CIB and has a similar spectrum. Template cleaning of the $C O B E / F I R A S$ data using only 3 spatial templates (the $158 \mu \mathrm{m}$ line from ionized carbon, the $21 \mathrm{~cm}$ line from neutral hydrogen, and the square of the $21 \mathrm{~cm}$ line intensity) successfully reduced a bright, complicated foreground by a factor of 10 to enable a statistically significant detection of the CIB intensity and spectrum (Fixsen et al. 1998). Furthermore, the ionized carbon template was derived from the FIRAS data itself, providing an example of a rich data set being used for internal cleaning. Detecting B-mode polarization in the CMB would require only modestly deeper cleaning, and would have the distinct advantage that the CMB frequency spectrum is known a priori.

Template cleaning has been demonstrated to reduce foreground emission by at least a factor of 10. The ultimate limit for this technique is not known. Given the large number of existing maps for various Galactic emission sources and the small noise penalty to be paid for fitting multiple templates, an interesting scenario would be to attempt a simultaneous fit of polarization data to as many templates as possible, including (as was done for the CIB) higher powers of individual templates. There will be additional templates available in the next five years, at low frequencies (5-20 GHz) from the C-Band All-Sky Survey (C-BASS), GEM-P (Barbosa et al. 2006), and COFE 


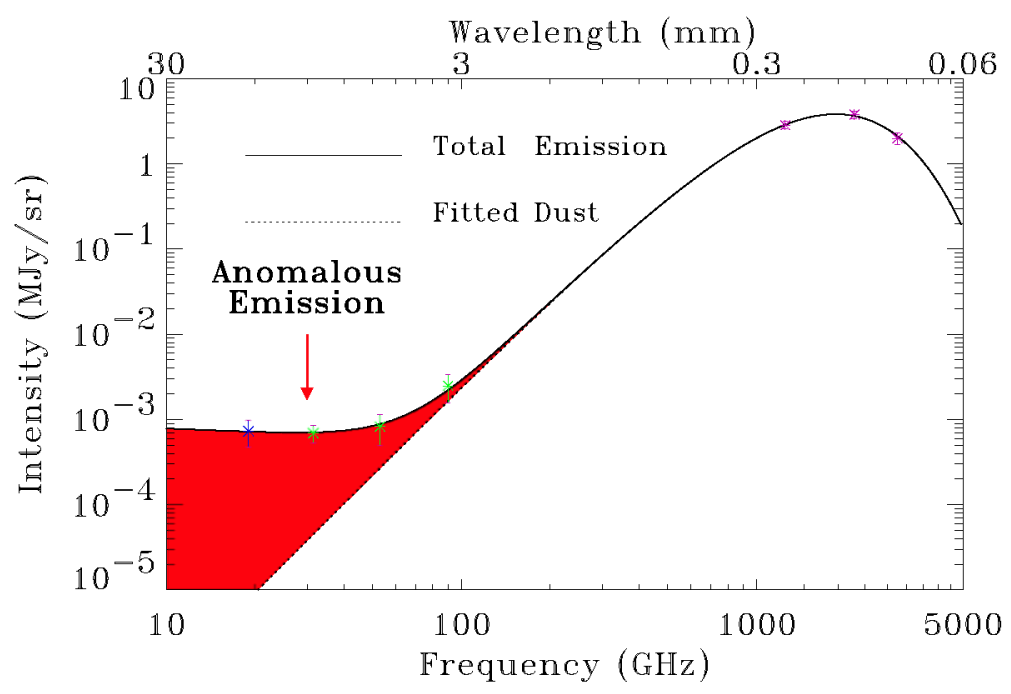

Figure 12: The frequency dependence of emission correlated with thermal dust shows an anomalous increase at centimeter wavelengths. Despite uncertainty in the physical origin of this correlated emission, it may efficiently be removed using template cleaning. From Kogut et al. (1996a).

(Leonardi et al. 2007), and at higher frequencies from the Planck satellite.

\subsection{Parametric methods}

Bayesian parameter estimation methods have begun to be applied to the problem of estimating the CMB signal, given observations of the sky at multiple frequencies. In contrast to template cleaning, which produces a cleaned map at each frequency, these methods use the multi-frequency information to estimate a single CMB map. The methods exploit our knowledge of the frequency dependence of different components. The signal is parameterized by a model, expressed as the sum of CMB and foreground components at each frequency. Current methods are split into two schemes. In the first approach the pixel Q and U CMB Stokes parameters are estimated in each pixel. In the second approach the CMB angular power spectrum is simultaneously estimated with the map parameters. In this framework, templates comprising the 'best-guess' for the emission of a particular foreground component can be included as priors. One of the main benefits of this method is that errors due to foreground uncertainty are rigorously propagated into the estimated CMB maps and power spectra. One of the drawbacks is that polarized components that are not in the parameterized model, or that are incorrectly described by the model, will not be properly accounted for. These methods are also typically computationally demanding.

\subsubsection{Estimation of CMB maps}

Eriksen et al. (2006), Gold et al. (2008), Dunkley et al. (2008b) and Stompor et al. (2008) describe a CMB component separation approach based on standard Bayesian parameter estimation techniques. A parametric model is used for each signal component (including CMB, synchrotron, and dust) and the probability distribution for the parameters is estimated. 
Using Bayes' theorem, the posterior probability is given by

$$
P(\theta \mid \mathbf{d}) \propto P(\mathbf{d} \mid \theta) P(\theta)=\mathcal{L}(\theta) P(\theta),
$$

where $\mathcal{L}(\theta)=P(\mathbf{d} \mid \theta)$ is the likelihood of the observed maps, and $P(\theta)$ is a prior for model parameters $\theta$. The likelihood is given by

$$
-2 \ln \mathcal{L}=\sum_{\nu}\left[\mathbf{d}_{\nu}-\mathbf{S}_{\nu}(\theta)\right]^{T} \mathbf{N}_{\nu}^{-1}\left[\mathbf{d}_{\nu^{\prime}}-\mathbf{S}_{\nu}(\theta)\right],
$$

where $\mathbf{N}_{\nu}$ is the noise covariance at each channel $\nu$, and the data contains some combination of I, Q, and $\mathrm{U}$ in map space. The model, $S_{\nu}(\theta)$, is minimally parameterized as the sum of CMB, synchrotron emission, and thermal dust emission components:

$$
\mathbf{S}_{\nu}=\boldsymbol{\alpha}_{c, \nu} \mathbf{A}_{c}+\boldsymbol{\alpha}_{s, \nu} \mathbf{A}_{s}+\boldsymbol{\alpha}_{d, \nu} \mathbf{A}_{d}
$$

For the CMB the coefficients are already known: the emission is black-body. For dust and synchrotron emission the coefficients are often constrained to give power law spectral indices, with $\alpha_{\nu}(\hat{n})=\left(\nu / \nu_{0}\right)^{\beta(\hat{n})}$. The amplitudes maps $\mathbf{A}_{i}$ are defined at pivot frequencies $\nu_{0}$. The goal is to estimate the CMB map $\mathbf{A}_{c}$ and its associated covariance.

The joint distribution for the amplitudes and spectral indices cannot be sampled directly, so in Eriksen et al. (2006) the MCMC Metropolis-Hastings algorithm (Metropolis et al. 1953; Christensen et al. 2001; Lewis \& Bridle 2002; Dunkley et al. 2005) is used to map out the the posterior probability distribution for the parameters $\theta$, using intensity data $(I)$. It has also been applied to polarization data (using $I, Q, U)$, in studies for the Planck satellite. In this case the noise is assumed to be diagonal, so each pixel is fit individually. The full parameter set, including component amplitudes and spectral indices, is initially fit pixel by pixel in low-resolution maps. This provides both best-fit values for each parameter and its associated uncertainty. The second sampling step is performed at higher resolution. All non-linear parameters are set at the best-fit values obtained in the lowresolution sampling, the parameter fields are smoothed spatially, and high-resolution amplitude maps for each component are then obtained analytically. The residual uncertainty in the measurement of the CMB emission in each pixel after foreground signals have been removed is estimated using the multiple bands. The technique is particularly appropriate given that Galactic emission anisotropy power dominates on large scales. The code 'FGFit' provides an implementation of this method and is also a useful forecasting tool. A similar method has also been implemented by Gold et al. (2008) and applied to the 5-yr WMAP temperature and polarization data.

An alternative method to estimate polarized CMB maps is described in Dunkley et al. (2008b). The principle is the same, but the sampling method is modified, with a Metropolis-within-Gibbs MCMC method used to map out the joint distribution $p(\theta \mid d)=p(\mathbf{A}, \boldsymbol{\alpha} \mid d)$. The Gibbs sampler is a special case of the Metropolis-Hastings MCMC algorithm. Specifically, suppose the target distribution of interest is $P(A, B)$, and we want to generate a joint sample $(A, B)$ from this distribution. This can be achieved by alternately sampling from the two conditional distributions,

$$
\begin{aligned}
& A^{i+1} \leftarrow P\left(A \mid B^{i}\right) \\
& B^{i+1} \leftarrow P\left(B \mid A^{i+1}\right) .
\end{aligned}
$$

Here, the left arrow indicates sampling from the distribution on the right hand side. In this method the parameter distribution is sliced into a conditional distribution for the amplitude maps, $p(\mathbf{A} \mid \boldsymbol{\beta}, \mathbf{d})$ for fixed non-linear parameters, and a distribution for the spectral indices $p(\boldsymbol{\beta} \mid \mathbf{A}, \mathbf{d})$, for fixed amplitude. The first distribution is a Gaussian and can be sampled directly. The second distribution is sampled using the Metropolis-Hastings algorithm. An advantage of this approach is that the noise matrix does not have to be assumed diagonal, and spectral indices can for example simultaneously 
be estimated in larger pixels than the amplitudes. Priors are imposed on the foreground emission. For example, for application to WMAP data, Dunkley et al. (2008b) assume that the spectral indices in $\mathrm{Q}$ and $\mathrm{U}$ are the same in a given pixel, and that in a map with 768 pixels, the spectral indices are only allowed to vary in $N_{i}=48$ larger pixels. In regions of low signal-to-noise priors are imposed on the synchrotron and dust spectral indices. A typical choice is a Gaussian prior of $\beta_{S}=-3.0 \pm 0.3$ on the synchrotron emission index, and $\beta_{d}=2 \pm 0.3$ (or broader) for the dust emission index.

While the methodology is different for these two methods, the principle is the same, and the resulting product is a CMB map and covariance matrix estimated from the marginalized posterior distribution. The likelihood of the estimated maps, given a theoretical angular power spectrum, can then be computed using the method described in Page et al. (2007). By varying only the tensor-toscalar ratio $r$, and calculating the likelihood at each value of $r$, this method can be used to estimate limits on the tensor-to-scalar ratio including foreground uncertainty.

\subsubsection{Estimation of CMB power spectra}

Rather than estimating the marginalized maps, and then estimating the CMB power spectrum, it is possible to simultaneously estimate both. Here the main goal is the joint posterior distribution $P\left(\mathbf{s}, C_{\ell}, \theta_{\mathrm{fg}} \mid \mathbf{d}\right)$, where $\mathbf{s}$ is the CMB sky map (denoted $\mathbf{A}_{c}$ in the previous sub-section), $C_{\ell}$ is the CMB power spectrum, $\theta_{\mathrm{fg}}$ denotes the set of foreground parameters, and $\mathbf{d}$ represents the data. From this joint distribution, one may extract any number of marginal distributions to obtain estimates for each parameter individually, of which perhaps the most important is the marginal power spectrum distribution $P\left(C_{\ell} \mid \mathbf{d}\right)$. From this one may extract cosmological parameters using a standard MCMC code such as CosmoMC.

The joint distribution $P\left(\mathbf{s}, C_{\ell}, \theta_{\mathrm{fg}} \mid \mathbf{d}\right)$ involves several millions of correlated parameters, and this poses a serious computational challenge. Brute force evaluation is impossible. However, in recent years a new Monte Carlo method based on the Gibbs sampling algorithm has been developed for precisely this purpose (Jewell et al. 2004; Wandelt et al. 2004; Eriksen et al. 2004; Larson et al. 2007; Eriksen et al. 2007b), and this allows the user to draw samples from the full joint distribution in a computationally efficient manner. Applications to real-world data include the analyses presented by O'Dwyer et al. (2004); Eriksen et al. (2007a,b).

In order to use the Gibbs sampler to sample from the joint CMB posterior, one must simply be able to sample from all corresponding conditional distributions,

$$
\begin{aligned}
C_{\ell}^{i+1} & \leftarrow P\left(C_{\ell} \mid \mathbf{s}^{i}, \theta_{\mathrm{fg}}^{i}, \mathbf{d}\right) \\
\mathbf{s}^{i+1} & \leftarrow P\left(\mathbf{s} \mid C_{\ell}^{i+1}, \theta_{\mathrm{fg}}^{i}, \mathbf{d}\right) \\
\theta_{\mathrm{fg}}^{i+1} & \leftarrow P\left(\theta_{\mathrm{fg}} \mid C_{\ell}^{i+1}, \mathbf{s}^{i+1}, \mathbf{d}\right) .
\end{aligned}
$$

Then, after some burn-in period, the set $\left(C_{\ell}^{i}, \mathrm{~s}^{i}, \theta_{\mathrm{fg}}^{i}\right)$ will be drawn from the desired joint distribution. Fortunately, sampling from each of these conditionals is straightforward, as the distribution for $C_{\ell}$ in Eqn. 17 is an inverse Wishart distribution, the distribution for $\mathbf{s}$ in Eqn. 18 is a multivariate Gaussian, and the distribution for $\theta_{\mathrm{fg}}$ is in general given by a $\chi^{2}$. All of these have well-known sampling algorithms (see, e.g., Eriksen et al. 2008a for a full review).

As in Section 3.2.1, the foreground model may be specified quite freely. For application to current data, the focus has been mainly on models with a small set of parametric foreground spectra with individual parameters for each pixel. Typical examples include power-law spectra $\left(S_{\nu}=A \nu^{\beta}\right.$; $A$ and $\beta$ free), one- or two-component thermal dust spectra, SZ spectra and arbitrary tabulated spectra. Additionally, Gaussian and/or uniform priors may be specified for each pixel. In the future, complementary sets of parameters describing a spatial power spectrum for each foreground component may be used, a feature which will be useful for probing the low signal-to-noise regime. 
A major advantage of the Gibbs sampling approach is, because of its conditional nature, its ability to integrate additional sources of uncertainty without modifying the existing framework. Three specific examples would be gain calibration uncertainties, beam uncertainties and noise estimation errors. By adding another conditional sampling step for each of these to the above Gibbs chain, the corresponding uncertainties would be seamlessly propagated through to all other parameters, without requiring any modifications to the main sampling scheme.

\subsection{Blind component separation}

In both the template and parametric methods, the foreground model is the sum of specific astrophysical components, and its construction relies on knowledge of their spatial behavior and/or frequency dependence. The goal of blind or semi-blind component separation methods is to separate the observed sky maps into a CMB component and a set of foreground components, with few assumptions about the physical behavior of those foreground components. Here we describe two methods, Internal Linear Combination (ILC) and Independent Component Analysis (ICA).

\subsubsection{Internal Linear Combination}

The ILC technique in spherical harmonic space was first described as a way to remove foregrounds from CMB anisotropies in Tegmark \& Efstathiou (1996). It was applied successfully to the WMAP one, three, and five-year temperature data (Hinshaw et al. 2003; Tegmark et al. 2003; de OliveiraCosta \& Tegmark 2006; Hinshaw et al. 2007; Gold et al. 2008), but it can also be applied to polarized data, using the $\mathrm{E}$ or B mode spherical harmonics (Amblard et al. 2007). The CMB signal is estimated as a linear combination of the observed $a_{\ell m}^{i}$ in each frequency band $i$,

$$
a_{\ell m}=\sum_{\text {freq }=i} w_{\ell}^{i} a_{\ell m}^{i}
$$

with weights $w_{i}$ chosen to minimize foreground contamination. For polarized CMB observations, the signal at each frequency can be decomposed as

$$
a_{\ell m}^{i}=c_{\ell m}+s_{\ell m}^{i}+d_{\ell m}^{i}+n_{\ell m}^{i},
$$

where $c, s, d$, and $n$ stand for the CMB, synchrotron, dust, and noise. The number of foregrounds can be increased, provided that the number of channels is also raised. To choose the weights that best extract the CMB signal, the power spectrum

$$
\left\langle\left|a_{\ell m}\right|^{2}\right\rangle=\mathbf{w}_{\ell}^{T} \mathcal{C}_{\ell} \mathbf{w}_{\ell}
$$

is minimized to optimally remove the foregrounds, where $\mathcal{C}_{\ell}^{i j}=\left\langle\left(a_{\ell m}^{i}\right)^{\dagger} a_{\ell m}^{j}\right\rangle$. The weights are constrained by $w_{\ell}^{T} \cdot \mathbf{e}=1$, where $\mathbf{e}$ is a column vector of all ones with length equal to the number of channels. This condition ensures that the CMB signal is retained. As derived in Tegmark et al. (2003), the minimized weights are given by

$$
\mathbf{w}_{\ell}=\frac{\mathcal{C}_{\ell}^{-1} \mathbf{e}}{e^{T} \mathcal{C}_{\ell}^{-1} e} .
$$

The estimated spherical harmonics are then used for cosmological analysis. The advantage of the ILC algorithm is that there are no assumptions made about the frequency dependence of the foregrounds. The algorithm maximizes the signal to noise of the recovered CMB, and accounts for differences in beam size between frequencies. The algorithm is computationally very fast, allowing multiple Monte-

Carlo simulations to be performed to propagate systematics and compute errors. A disadvantage of 
the method is that the foregrounds are assumed to have a spatially invariant frequency behavior, which is not physically realistic. This can be relaxed by splitting the full sky maps into several areas. Further drawbacks are that the total power spectrum is minimized rather than just the foreground power, and it is difficult to rigorously propagate errors into the estimated CMB maps.

\subsubsection{Independent Component Analysis}

As with the ILC method, ICA components are linear combinations of the input sky maps, but the weights are chosen using an alternative method. Two examples are 'altICA' and Spectral Matching ICA (SMICA).

The altICA method is based on an algorithm by Hyvarinen (1999), and is described in Maino et al. $(2002)^{4}$. The weights given to the sky maps for each component are estimated by maximizing the non-Gaussianity of the component maps. This idea is based on the central limit theorem, which states that a variable that is a mixture of independent variables is more Gaussian than the original ones. By transforming the observed sky maps into a set of components such that the Gaussianity of the variables is reduced, the resulting maps should be more independent. The algorithm has been implemented as a CMB cleaning procedure, because the CMB and diffuse foregrounds are expected to be statistically independent. However, the non-Gaussianity of a given foreground component may vary spatially, so this decomposition is not necessarily astrophysically motivated.

In this method, the degree of non Gaussianity is quantified by the neg-entropy statistic, which is more robust than many other statistics in the presence of noise. The neg-entropy for a map $\mathbf{y}$ (containing $I, Q$ or $U$, for a given set of weights), is defined as

$$
\operatorname{neg}-\operatorname{entropy}(\mathbf{y})=H\left(\mathbf{y}_{G}\right)-H(\mathbf{y}),
$$

where $H(\mathbf{y})=-\int p(\mathbf{y}) \log p(\mathbf{y}) d \mathbf{y}$ is the entropy associated with the distribution $p(\mathbf{y})$, and $\mathbf{y}_{G}$ is a Gaussian variable with the same covariance matrix as $\mathbf{y}$. This method can be extended to include a priori knowledge of the frequency scaling of one or more components. The method has been applied to real and simulated intensity data (including BEAST, COBE and WMAP) and simulated polarization data (see Maino et al. (2007) and references therein). The performance relies on the assumption of statistical independence for CMB and foregrounds, and operates most successfully with high resolution data.

In the SMICA method (Delabrouille et al. 2003; Cardoso et al. 2008), the CMB and foreground power spectra are estimated using a parametric model of the angular power spectra of the components. For application to Planck simulations (Cardoso et al. 2008), the angular spectra and cross-spectra, $C_{\ell}^{i j}$, measured at $N_{\nu}$ frequencies, are parameterized as the sum of CMB, foregrounds, and noise:

$$
C_{\ell}^{i j}=C_{\ell}^{\mathrm{CMB}, i j}+F_{\ell}^{i j}+N_{\ell}^{i j},
$$

with $C_{\ell}^{i j}=1 /(2 \ell+1) \sum_{m}\left(a_{l m}^{i}\right)^{\dagger} a_{l m}^{j}$ for frequencies $i$ and $j$. In practice the spectra are binned in $N_{q}$ bins in $\ell$, to give spectra $C_{q}^{i j}$. The foreground model is parameterized by $D$ possibly correlated angular power spectrum templates, with

$$
F_{q}=A^{T} P_{q} A
$$

Here $\mathrm{A}$ is a matrix of size $D \times N_{\nu}$ and $P_{q}$ is a symmetric matrix of size $D \times D$. This gives a total of $N_{\nu} \times D+N_{q} \times D(D+1) / 2$ foreground parameters. SMICA allows foregrounds to be correlated, which is useful for modeling Galactic components that are naturally correlated by the geometry of the Galactic plane and the effect of the Galactic magnetic field. To capture the complexity of

\footnotetext{
${ }^{4}$ The altICA algorithm is the result of a study supported by NASA LTSA Grant NNG04CG90G.
} 
the Galactic emission in polarization, at least two templates are needed for synchrotron and dust emission. In practice, the number of components required to capture the physical behavior depends on the frequency range and noise levels of the experiment. To study CMBPol missions, $D=4$ templates are used for the Galactic emission, which captures synchrotron and dust emission, and corrections due to variable emission laws. This parameterization of the foregrounds is considered 'semi-blind' as it allows only a limited set of foreground spectra.

The model for the binned CMB angular spectrum, $C_{q}^{\mathrm{CMB}}$, is parameterized by cosmological parameters including the tensor-to-scalar ratio. Prior knowledge can be introduced in the modeling, for example by fixing the B-mode power spectrum of the lensed CMB to the known value. The noise spectra $N_{q}^{i j}$ are considered as additional components in each channel. Noise levels are either assumed known, in which case the noise spectra are fixed, or else are estimated simultanously with the foreground and CMB spectra. This model is fit to the measured angular spectra of multifrequency sky maps. Since the spectra of each foreground component are expected to vary spatially, the fit is done in three spatial regions using apodized masks, to cut out the sharp features of the Galactic plane. The model parameters, $\theta$, are estimated by minimizing a mismatch statistic, $\phi$, between the model and the data:

$$
\phi(\theta)=\sum_{q=1}^{N_{q}} \frac{w_{q}}{2}\left[\operatorname{tr}\left(\mathrm{C}_{\mathrm{q}}(\theta)^{-1} \hat{\mathrm{C}}_{\mathrm{q}}\right)-\log \operatorname{det}\left(\mathrm{C}_{\mathrm{q}}(\theta)^{-1} \hat{\mathrm{C}}_{\mathrm{q}}\right)-\mathrm{m}\right],
$$

where $\mathbf{C}_{q}(\theta)$ and $\hat{\mathbf{C}}_{q}$ are the binned spectra of the model and data in bin $q$, and $w_{q}$ is the effective number of modes in the bin. The errors on the CMB spectrum are estimated using the Fisher matrix.

This method has been used for CMB cleaning and power spectrum estimation for Planck simulations (Leach et al. 2008). It has also been used to estimate the temperature power spectrum of the CMB for WMAP first year data (Patanchon et al. 2005) and for Archeops data (Tristram et al. 2005). To estimate the tensor-to-scalar ratio in the presence of foreground emission, one can assume a fixed shape of the CMB B-mode angular power spectrum, and then vary $r$ in the parameter fit by varying the overall amplitude. This method produces estimates of the power spectra of each component. Maps of the CMB and of each foreground component can be estimated by Wiener filtering the observed multi-frequency maps, using the angular power spectrum of the best-fit model as the filter.

\subsection{Discussion}

These three classes of methods have all been applied to observed or simulated polarization data, and have different advantages and drawbacks. The template-cleaning method is simple and has been demonstrated to work effectively with current data. We do not yet have a good template for the polarized dust, or for low polarized components such as free-free, but multi-frequency information from CMBPol should be able to provide such templates. It is possible to propagate errors due to foreground uncertainty into the template-cleaned maps, but the framework for doing so is less well-defined than for some other methods. The parametric sampling methods are ideal for propagating errors due to foreground uncertainty in a fully Bayesian framework into both CMB maps and estimated power spectra. Additional observations can also be included as priors, such that all the information we have about the Galactic emission can be used. These methods could be biased if the physical model does not match the true sky, but various models can be tested to establish the most suitable one. They can also be numerically intensive. The blind component separation methods described here are quick to perform, and have the advantage of not assuming a particular physical source for the foregrounds. However neither do they take advantage of the fact that we do have useful knowledge of the emission processes, and can make unphysical assumptions about the 


\begin{tabular}{ccc}
\hline $\begin{array}{c}\text { Frequency } \\
\text { GHz }\end{array}$ & $\begin{array}{c}\text { FWHM } \\
\operatorname{arcmin}\end{array}$ & $\begin{array}{c}\Delta \mathrm{T}(\mathrm{pol}) \\
\mu \mathrm{K} / \operatorname{arcmin}\end{array}$ \\
\hline 30 & 26 & 19.2 \\
45 & 17 & 8.3 \\
70 & 11 & 4.2 \\
100 & 8 & 3.2 \\
150 & 5 & 3.1 \\
220 & 3.5 & 4.8 \\
340 & 2.3 & 21.6 \\
\hline
\end{tabular}

Table 3: Specifications for a CMBPol $2 \mathrm{~m}$ mission, for frequencies below $350 \mathrm{GHz}$. The noise limits $\Delta T$ are polarized limits for $\mathrm{Q}$ and $\mathrm{U}$.

foregrounds, for example including limited spatial variation of spectral indices. Cleaning currently observed polarization maps from WMAP has required methods operating in pixel space, so power spectrum methods may need further testing with real data.

At the current time the community has tools ready to apply to polarization data with the sensitivity of a CMBPol satellite. Data from Planck and ground-based experiments will allow more extensive testing and comparisons between these methods, but we are ready now to tackle a CMBPol data set. In the event of a gravitational wave detection it is clear that having more than one method available will be important for cross-checking results.

\section{Numerical forecasts}

In this section we attempt to estimate whether a detection of a gravitational wave signal with $r=0.01$ is possible for a realistic example mission. The specifications for this mission are given in Table 3 and have arisen from CMBPol studies focused on systematic effects and technological capabilities. We also consider how to optimize the allocation of detectors with frequency for such a mission.

\subsection{Constraints on the CMB signal}

We consider two approaches to obtain forecasts on the B-mode CMB signal: a Fisher matrix method assuming that the foregrounds can be cleaned to a specified level, and a simulation approach, where we apply cleaning methods described in Section 3 to simulated maps, to estimate the CMB signal. For simplicity we will focus on the possible detection of a primordial signal with tensor-to-scalar ratio $r=0.01$. This level is picked as a target that CMBPol should aim to reach, based on theoretical arguments discussed in Baumann et al. (2008b). In this section all estimates are derived using the experimental specifications given in Table 3.

Note that in addition to statistical and systematic uncertainties estimated in the approaches detailed below, the detection significance of a B-mode signal is also affected by cosmic variance. The error bars on the power spectrum, $C_{\ell}$, with noise spectrum $N_{\ell}$, are given approximately by

$$
\sigma\left(C_{\ell}\right)=\sqrt{\frac{2}{(2 l+1) f_{s k y}}}\left(C_{\ell}+N_{\ell}\right) .
$$

As $r$ tends to zero, the cosmic variance term also goes to zero. For non-zero $r$, cosmic variance will therefore affect the confidence interval. This means that a simple detection of B-modes can be 
confirmed with higher confidence than can a measurement of a specific small-amplitude signal such as $r=0.01$.

\subsubsection{Fisher matrix approach}

The Fisher matrix approach described in Verde et al. (2006) is used for forecasting errors on measured cosmological parameters. In this analysis the lensing contamination in the B-mode signal is accounted for by assuming that the lensing power spectrum can be accurately estimated, but not removed from the maps, such that it acts as an additional Gaussian noise term. To include foregrounds, the parameterization given in Section 2.5 is adopted for the total foreground signal, with the synchrotron given by Eqn. 6. The dust model is given by Eqn. 7 with $A_{d}=0.04 \mu K^{2}$ at $\ell=10$, and a scale dependence $m=-0.5$, corresponding to an observed dust polarization fraction of $\sim 4 \%$. At scales $\ell \gtrsim 30$ this model matches the predicted spectrum from the Planck Sky Model. At larger scales the power spectrum of the simulated map flattens, which is not captured by this power law behavior ${ }^{5}$.

For a description of the method, see Appendix C in Baumann et al. (2008b) and Verde et al. (2006). The method assumes that at each frequency the angular power spectrum of the foregrounds can be cleaned to a given residual level. The lowest and highest frequencies are discarded, assuming that they will act as foreground templates. Residual levels of both $30 \%$ and $10 \%$ of the total amplitude (10\% and 1\% in power) are considered, shown in Figure 13 for a $10 \%$ residual at $90 \mathrm{GHz}$. These are chosen to represent a pessimistic and realistic case. Experience with previous experiments suggests that reducing the foreground amplitude by a factor of 10 (or more) should be possible, corresponding to the realistic case. The pessimistic case allows for poorer foreground cleaning, or a higher total foreground level than given by the model.

Considering only the large-scale power, with $\ell<15$, a model with $r=0.01$ can be detected at almost $7 \sigma$ in the absence of foregrounds, and assuming all other parameters are known. The error, $\sigma_{r}=0.0015$, is given in Table 4, and is unmarginalized, so all other cosmological parameters are assumed known. With 10\% residual foreground amplitude, the primordial signal is undetectable according to this method, with $\sigma_{r}=0.014$. However, this should be considered an overly pessimistic scenario, as improved cleaning is likely possible, and more importantly the large-scale foreground power is likely over-estimated, as the spectrum of the dust intensity flattens at large scales rather than following a pure power law. Including smaller scales, prospects are much improved. In the absence of foregrounds the $r=0.01$ signal is detected at $20 \sigma$ with $\sigma_{r}=0.00046^{6}$, and this is only decreased to $14 \sigma$ with pessimistic residual foregrounds. With $10 \%$ residual foregrounds, the estimated degradation of the error on $r$ is small. The analysis, reported in Baumann et al. (2008b), also shows that information about the scale dependence of the tensors can be extracted by observing a range of scales, with forecasted errors on the tensor spectral index, $n_{t}$, of $\sigma\left(n_{t}\right) \sim 0.1$.

These forecasts should be interpreted with care. Some of the assumptions made in producing these forecasts, for example, whether the lensing treatment is realistic (discussed further in Smith et al. (2008)), and the assumed polarized dust levels at $\ell=100$, need to be tested and may affect the resulting error bars. The estimated errors also assume that there is no effect of leakage of power from $\mathrm{E}$ to $\mathrm{B}$ modes. By using a large fraction of the sky, the errors on the measured polarization

\footnotetext{
${ }^{5}$ This dust model corresponds to dust 'Model A' reported in Baumann et al. (2008b). An additional 'Model B' is also considered in Baumann et al. (2008b), with $A_{d}=0.002 \mu K^{2}$ at $\ell=10$, with scale dependence $m=0.6$. The scale dependence of the dust is expected to lie within the $-0.6<m<0.6$ range considered in the two models. For simplicity we report only on Model A in this study, as it most closely matches simulated maps.

${ }^{6}$ This limit depends somewhat on assumptions made in the forecast. For example, in the absence of lensing noise, limits of $\sigma_{r}=0.0002$ are obtained for $f_{s k y}=0.87$.
} 


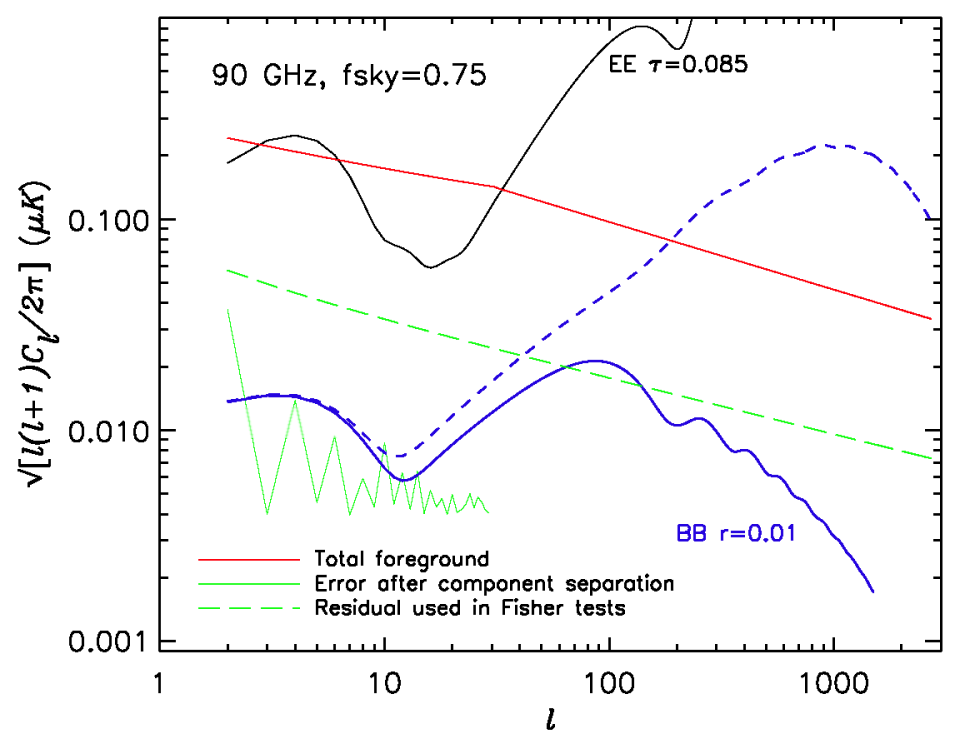

Figure 13: Comparison of CMB signals (E-mode - solid black; B-mode from inflation solid blue,; total B-mode, including lensing - short dash), and an estimate of the residual foreground signal. Foreground spectra (for synchrotron plus dust) are given at $90 \mathrm{GHz}$ for the cleanest $75 \%$ of the sky. The red line shows the total foreground signal, for simulated maps with mean dust polarization fraction $1.5 \%$. Using a component separation technique on low resolution simulated maps gives an instrument noise plus foreground residual power spectrum (green), that is about $5 \%$ of the expected foreground at large scales. The green dash line is the power spectrum of foreground residuals assumed in the Fisher matrix analysis, where the mean dust polarization fraction is $5 \%$. This likely over-estimates the residual foreground power at low $\ell$.

will vary spatially when foreground uncertainty is included, resulting in additional contamination of the B-mode signal from E-mode leakage. Amarie et al. (2005) approach this problem by masking out all regions of sky where the foregrounds exceed some fraction of the CMB signal. This leads to larger sky cuts than the $20 \%$ cut used in this analysis, and larger errors than obtained here for the $\ell<150$ case. We address this issue further in the next sub-section.

\subsubsection{Component separation with simulated maps}

An alternative approach is to apply foreground removal methods to simulated maps, in order to forecast the accuracy to which the CMB signal is recovered. In this initial study we test the parametric and blind component separation methods, as numerical codes already exist to do so. We do not consider template-cleaned forecasts here, leaving this approach for future investigation.

\section{Parametric methods:}

Simulations at each frequency for the CMBPol mission specified in Table 3 are formed using 


\begin{tabular}{ccccc}
\hline Method & $\begin{array}{c}\text { Average dust } \\
\text { pol fraction (\%) }\end{array}$ & Description & $\ell<15$ & $\ell<150$ \\
\hline Fisher & 0 & No foregrounds & 0.0015 & 0.00046 \\
Fisher & 5 & $10 \%$ residual & 0.014 & 0.00052 \\
& & & & \\
Parametric & 1 & Fixed spectral indices & 0.0015 & 0.0005 \\
Parametric & 1 & Power-law indices & 0.0025 & - \\
Parametric & 5 & Power-law indices & 0.003 & - \\
Blind & 4 & SMICA & - & 0.00055 \\
& & & & \\
\hline
\end{tabular}

Table 4: The forecast $1 \sigma$ uncertainties on the tensor-to-scalar ratio for fiducial model $r=0.01$, assuming other cosmological parameters are known, for a set of foreground assumptions. The 'Fisher' and 'Blind' tests include a residual noise signal from lensing. The 'Parametric' tests ignore lensing as a contaminant, a poor approximation at $\ell>15$. For $\ell<15$, the errors in the presence of foregrounds from the Fisher matrix test are higher than those obtained by other methods, as the assumed residual foregrounds are also higher at large scales.

HEALPix with $N_{\text {side }}=16$ and $N_{\text {side }}=128$, using the following model:

$$
\mathbf{m}(\nu)=\mathbf{A}_{\text {synch }}(\nu)+\mathbf{A}_{\text {dust }}(\nu)+f(\nu) \mathbf{A}_{\mathrm{cmb}}+f(\nu) \mathbf{n}(\nu),
$$

wherewhere $f(\nu)$ converts thermodynamic to antenna temperature. The maps are formed using Eqn. 5 for the synchrotron, and Eqn. 8 for the dust. Since they include the synchrotron and dust geometric suppression factors, the average dust polarization is $1 \%$ in the fiducial case (current PSM maps have average 5\% polarization fraction; we also consider maps with this increased dust level). Unlensed CMB maps are generated using the HEALPix synfast routine, for $r=0.01$ with all other parameters fixed to the best-fit $\Lambda$ CDM parameters estimated from the WMAP five-year data Komatsu et al. (2008). The $N_{\text {side }}=16(128)$ maps are smoothed with a 7 (1.5) degree beam. Gaussian noise is added uniformly over the sky, and $1 / f$ noise is ignored. In Figure 13 we plot the angular power spectrum of the total foreground signal in these maps at $90 \mathrm{GHz}$. Part of the goal of performing the component separation is to assess the size of the errors on the estimated CMB maps, or power spectra, compared to the total signal.

Marginalized CMB maps: We apply the parametric fitting methods described in Section 3.2 to the simulated maps, to estimate the foreground-marginalized errors on the observed CMB signal in map space. For a single random individual pixel outside the Galactic plane, the parameters of the model are sampled using the MCMC method described in Sec 3.2.1 ${ }^{7}$, and the marginalized error and bias on the CMB pixel amplitude are reported in Table 5, averaged over 5000 simulations. The errors are inflated about seven times compared to the foreground-free case. This level depends somewhat on the size of pixel in which the spectral indices are allowed to take unique values, in this case two degrees. We also estimate full marginalized CMB maps for this model ${ }^{8}$. The marginalized error map for the Q Stokes parameter is shown in Figure 14. In this case the spectral indices are fit in

\footnotetext{
${ }^{7}$ Using the FGFit code described in Eriksen et al. (2006).

${ }^{8}$ Using the method and code described in Dunkley et al. (2008b).
} 


\begin{tabular}{cccc}
\hline Case & Error $(\mathrm{nK})$ & Case & Error (nK) \\
\hline No foregrounds & 8 & Increased synchrotron & 79 \\
Fiducial model & 58 & Increased dust & 60 \\
Extended model & 58 & Increased dust + synchrotron & 81 \\
\hline
\end{tabular}

Table 5: Expected per-pixel sensitivities in polarization in $4 \times 4$ degree pixels in $n K$, including foreground degradation, for the $2 \mathrm{~m} \mathrm{CMBPol}$ example mission. The 'extended' model has a break in the synchrotron index, and low levels of polarized free-free and spinning dust emission. 'Increased' means a three-fold increase in amplitude.

pixels of side 3.7 degrees, so errors are slightly reduced compared to the 2 degree pixels used in Table 5. The errors should be compared to the co-added noise level of $8 \mathrm{nK}$ per pixel, in the absence of foregrounds. Increased errors, up to a maximum of $50 \mathrm{nK}$, are due to foregound marginalization. We then ask how big the marginalized errors on the CMB signal are compared to the total foreground signal. To do this we take the marginalized error maps and compute their angular power spectra, masking $25 \%$ of the sky using the WMAP P06 mask. This spectrum is shown in Figure 13, and is comparable to the CMB spectrum for $r=0.01$. The amplitude is about $5 \%$ of the total foreground signal at $90 \mathrm{GHz}$. This estimate may be over-optimistic if the true sky has more complicated spectral behavior and additional low polarization components that were not included in this test. However, in this test we allowed each pixel of side 3.7 degrees to have unique synchrotron and dust spectral index parameters. If we are only interested in a broad-band detection of the B-mode signal, larger pixels may be averaged together and could lead to lower residual error levels.

Since there is about an order of magnitude uncertainty in the dust amplitude, we repeat the tests with varied foreground levels. For the case of an individual pixel, if we inflate the synchrotron amplitude three times, this inflates the errors by a further $50 \%$, but a similar increase in the dust levels, where we are far less certain, has a smaller effect. If the simulation is modified to have a curvature in the spectral index, together with a $1 \%$ polarized free-free and $2 \%$ polarized spinning dust component with random polarization angles, the marginalized error is negligibly different from the fiducial case. In this case the model is sufficiently flexible that the 'synchrotron' component absorbs the additional polarized components, with an effective spectral index that is the weighted sum of the components. In future studies it will be useful to explore simulations that deviate further from the model, to test whether there is a significant bias in the recovered CMB power when the model fails to match the simulated data.

To estimate the CMB power in these simulated maps, we use the Gibbs sampling method described in Section 3.2.2, using the Commander numerical code (Eriksen et al. 2007a) ${ }^{9}$. Table 4 shows $1 \sigma$ errors on $r$, assuming other parameters are perfectly measured. When the foreground spectral indices are known perfectly, the tensor-to-scalar ratio is recovered with $r=0.0106 \pm 0.0017$ for $\ell<15$, and $r=0.0101 \pm 0.0005$ for $\ell<150$. These error bars are similar to those obtained in the Fisher matrix tests in the absence of foregrounds, where lensing noise is also included. These errors are too optimistic however, as in reality the synchrotron and dust spectral indices are uncertain and should be marginalized over. However, this provides a test of the method and shows that in this simple case the recovered signal is not significantly biased. It also indicates that regions of the sky where the foregrounds are high can still be used to estimate the CMB power: rather than masking out pixels as in Amarie et al. (2005), the errors are inflated in regions where foreground uncertainty

\footnotetext{
${ }^{9}$ We cross check results for low resolution maps by computing the exact likelihood of the marginalized maps, using the method in Section 3.2.1. These two methods should give the same results for any given set of simulated maps, although the implementation is different.
} 
is high, and propagated into the likelihood.

A more realistic case includes the possible variation of synchrotron and dust spectral indices in each pixel. For pixels of side 4 degrees, and scales $\ell<15$, the error bar on $r$ is increased to $\sigma_{r}=0.0025$, indicating only a $4 \sigma$ detection of the primordial signal. This is increased to $\sigma_{r}=0.003$ when the dust component in the simulated maps is made three times larger (corresponding to $4 \%$ polarization fraction). The limits may worsen if additional freedom in the foreground model is allowed (e.g. polarized free-free emission, a two-component dust power law, or a synchrotron spectral curvature), and if the dust is more then $5 \%$ polarized, although additional observations may provide priors or templates that would improve limits. Observations from the Planck satellite and ground based experiments will help to guide such choices for future preparatory studies. The application of this spectral index marginalization to the higher resolution maps, using $\ell<150$, was not calculated in this initial study, but is an obvious next step.

\section{Blind separation methods:}

The ILC method in spherical harmonic space described in Section 3.3.1 and the SMICA component separation method described in Section 3.3.2 have also been applied to simulated maps.

For tests with SMICA, alternative maps to the fiducial simulation were used, as part of a Planck preparatory study. The results can be compared with others considered in this Section, as the maps are very similar to our 'Increased dust' model, with dust that is $4 \%$ polarized on average. The SMICA method assumes perfect knowledge of all cosmological parameters except $r$, the latter assumed to be measured only from B-modes. For the mission specifications given in Table 3, limits of $\sigma_{r}=0.00055$ are found for $\ell<150$ (Betoule et al. 2008) for a model with $r=0.01$. This includes additional noise from the lensing signal, which when ignored leads to a limit of $\sigma_{r}=0.00046$. Point sources are assumed to have a negligible impact on limits on $r$. These are similar to the limits found using the Fisher matrix method. When an additional $500 \mathrm{GHz}$ channel is included, estimated limits improve to $\sigma_{r}=0.0003$, indicating that this longer lever arm could provide useful additional information. However, it is possible that extrapolating from these high frequencies can introduce errors due to incorrect modeling assumptions. The numerical experiment with SMICA indicates that even with some complexity in the foreground modeling, estimation of B modes may be performed satisfactorily on a significant fraction of the sky, supporting large sky coverage for the CMBPol mission.

Using the ILC method, rather optimistic levels of detection are obtained. Confidence levels on a model with $r=0.01$ were not considered, but the $99 \%$ confidence level detection of $r$ for fiducial $r=0$ is 0.0002 for $\ell<8$, and 0.0004 for $50<\ell<130$ in the absence of lensing. ${ }^{10}$ This would imply foreground cleaning to better than $\sim 1 \%$ in amplitude at large scales and better than $5 \%$ at intermediate scales, which is likely difficult to achieve in practice.

\subsection{Optimal use of focal plane}

Variables that drive experimental design include frequency range, bandwidth, and number of channels. To investigate the optimal configuration one requires either an analytic form for the errors, or a component separation code that can be run quickly. For this work, we employ the parametric method described in Section 3.2.1 to perform component separation for a single pixel, repeated many times with different $\mathrm{CMB}$ and noise realizations. ${ }^{11}$ The averaged best-fit value for the CMB amplitude, and its marginalized error, are estimated. The error is then used as the statistic to compare the relative performance of different experimental specifications. In principle the number of channels, positioning in frequency space of the channels, and angular resolution can be varied.

\footnotetext{
${ }^{10}$ With lensing as a contaminant, this increases to $r<0.001$ for $\ell<8$ and $r<0.008$ for $50<\ell<130$.

${ }^{11}$ FGFIT_pix was designed exactly for this purpose, with input files for multiple experimental design and sky models. The experiment file contains the frequency and noise level for each channel.
} 


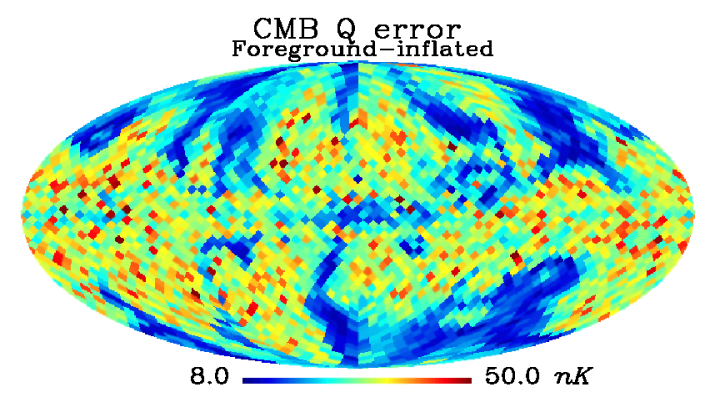

Figure 14: Marginalized errors for a CMB Q Stokes map, using the CMBPol mission specified in Table 3 and the fiducial foreground model. In the absence of foregrounds the noise level would be $8 \mathrm{nK}$ in each pixel. The errors are increased where the signal is most uncertain due to foreground contamination.

Here we perform a limited study for a fixed number of ten channels, chosen to allow for at least three parameters in each pixel for both synchrotron and thermal dust, and two for additional low-polarization components. The channels are spaced at logarithmic intervals in frequency, with the number of detectors at each channel varied such that the total signal-to-noise ratio ('total' is CMB plus foregrounds) is kept constant. The maximum and minimum frequencies are then varied, and the error on the CMB amplitude estimated. The foregrounds are assumed to have power law spectral indices for the parameterized model. The preferred minimum frequency is found to be at $\sim 40 \pm 5 \mathrm{GHz}$, with a poorly constrained maximum at $\sim 200-300 \mathrm{GHz}$. This spans a similar frequency range as proposed in the initial mission design, although has more channels. A number of different assumptions are investigated for the distribution of detectors at each frequency, using different scalings for sensitivities as a function of frequency. They give similar results for the optimal frequency range.

To use these tests to guide mission design, further investigation of the poorly constrained upper frequency limit would be useful. An extended parameterization for the foreground model could also be explored, to test how the optimal range is affected by the assumption of power law behavior. The number of channels can be explored with a similar tool, but was not considered in this initial study. It is clear that more channels will help to remove the foregrounds effectively and break degeneracies between spectra of different emission mechanisms. Sparsely placed frequency channels lead to uncertainty in the curvature of the foreground frequency spectra. However, there is always a trade-off between increased sensitivity at each frequency, and increased number of channels.

If we ignore the effects of lensing there is little motivation to go to higher resolution than $\approx 1$ degree. However, optimization for lensing should be considered in order to extract the gravitational wave signal at smaller scales where the foregrounds are lower than at the lowest multipoles. See the companion lensing report by Smith et al. (2008) for more details.

\subsection{Future tests}

The forecasts presented here represent an initial investigation into the CMBPol mission capabilities. Work remains to be done to make concrete forecasts, but we will be in a better position as more data is obtained from the Planck satellite and ground-based experiments. Further preparatory work for a CMBPol mission could include:

(i) Bias and modeling: So far we have examined cases where the simulated maps are correctly described by the models. In a realistic scenario, the model will not perfectly capture the observed two-dimensional emission. It will be useful to determine how many parameters we need to fit the 
true sky 'well enough', and whether an incorrect model leads to significant bias in the estimate of the CMB signal.

(ii) Convergence of estimates: Different methods exist for forecasting the constraining abilities of the CMBPol mission. Consensus of the effect of foregrounds on the estimated B-mode signal is important in the planning of the mission, and will likely improve with better observations. However, not all methods will clean the maps equally well, so we would not necessarily expect to achieve the same errors on the CMB signal from all component separation methods. We plan to continue with the program we have begun, to compare the estimated residual noise-plus-foreground levels for various methods over the $\ell<150$ range, accounting for the lensing signal as a source of noise in simulations. These would include the Fisher methods, parametric and blind separation methods described here, as well as template cleaning applied to forecasting.

(iii) Optimizing the experiment: The community has tools that can be applied to experimental optimization. In particular the parametric fitting employed in the 'FGfit' code allows for easy comparison of the CMB errors in a pixel, marginalized over foregrounds, for different experimental set-ups. Other methods, including template cleaning and ILC component separation, can be used for alternative investigations. Further exploration with these tools will be useful, including tests for the optimal number of channels, as well as variation of the complexity of the model. One can also test the effect of varying the experimental sensitivity, to determine the noise level at which the observations become foreground dominated.

\section{Summary}

Polarized Galactic emission at microwave frequencies is dominated by two components, synchrotron emission and thermal dust emission. The synchrotron emission has been well measured on large angular scales at $23 \mathrm{GHz}$ by WMAP. Our knowledge of polarized dust emission is relatively poor, particularly in the low surface brightness regions out of the Galactic plane targeted for CMB observations. It will soon be better characterized by the Planck satellite, due for launch 2009, which will measure polarized emission with a spatial resolution $<30$ arcmin at $30-350 \mathrm{GHz}$. With the assumption of a polarized dust fraction of $1-10 \%$, the total foreground emission is expected to be roughly a factor of eight brighter, on angular scales of two degrees over $75 \%$ of the sky, than a gravitational wave signal with tensor-to-scalar ratio $r=0.01$. There may also be small polarization contributions from free-free emission, spinning dust emission, or other 'anomalous' components that have not yet been detected.

Past experience working on analogous problems suggests that the challenge of dealing with significant foreground components is not insurmountable, and has been addressed successfully with previous experiments such as FIRAS. In this case the CIB foregrounds were reduced by a factor of ten to extract the signal, comparable to the cleaning required of polarized Galactic foregrounds for the CMB. Although past performance is no guarantee of future success, we have consistently been able to clean foregrounds to within a factor of a few times the uncertainties of the raw measurements. As a community we have a set of tools ready for performing component separation and estimating the CMB signal from observed sky maps. These could be applied today to CMBPol data, and will be significantly improved and tested with data from Planck and from ground and balloon-based experiments during the next five years. There is some variation in predicted performance for a specified CMBPol mission, so the refinement process with upcoming experiments will be useful for understanding why some methods perform better than others, and whether this depends on details in our simulations.

Despite the good prospects for cleaning foregrounds, such prospects rely sensitively on experimental design. Observations over a wide range of angular scales should be planned in order to mitigate anticipated residual Galactic contamination on the largest angular scales $(\ell<15)$. Multi- 
ple channels are needed to characterize and remove the foregrounds. Although there are only two dominant polarized components, they are unlikely to have spectral indices that can be modeled accurately as power laws. This means that at least three channels per component should be included. Since there may also be additional low-polarization components, at least ten channels spaced logarithmically in the range $\sim 40<\nu<200-300 \mathrm{GHz}$ would be advantageous, although further studies should be undertaken to explore this in more detail. Extrapolating from very low and high frequencies can introduce modeling errors, so frequencies below $\sim 300 \mathrm{GHz}$ are more easily used for foreground subtraction.

Current methods and simulations indicate that we can clean Galactic foregrounds from maps of the polarized sky to at least the $5-10 \%$ level. There are therefore good prospects that, for a realistic CMBPol mission design, a gravitational wave signal with $r=0.01$ could be detected at more than $10 \sigma$. Successfully disentangling this signal from the emission of our own Galaxy will provide rich rewards, as a detection of primordial B-modes would open up a new window on the earliest moments in the universe.

This research was partly funded by NASA Mission Concept Study award NNX08AT71G S01. We thank Scott Dodelson for coordination of the CMBPol Theory and Foregrounds workshop and proceedings, and acknowledge the organizational work of the Primordial Polarization Program Definition Team. We thank David Spergel and Alex Lazarian for useful comments. JD acknowledges support from an RCUK fellowship. GR is supported by the US Planck Project, which is funded by the NASA Science Mission Directorate. We thank the WMAP team for making maps available on LAMBDA, and acknowledge the use of the Planck Sky Model, developed by the Component Separation Working Group (WG2) of the Planck Collaboration. We acknowledge use of the HEALPix, PolSpice, CAMB, and CMBFAST packages.

\section{References}

Aitken, D. K. 1996, in ASP Conf. Ser. 97, Polarimetry of the Interstellar Medium, ed. W. G. Roberge \& D. C. B. Whittet, 225

Ali-Haïmoud, Y., Hirata, C. M., \& Dickinson, C. 2008, ArXiv e-prints, 0812.2904

Amarie, M., Hirata, C., \& Seljak, U. 2005, Phys. Rev. A, 72, 123006, arXiv:astro-ph/0508293

Amblard, A., Cooray, A., \& Kaplinghat, M. 2007, Phys. Rev. A, 75, 083508, astro-ph/0610829

Barbosa, D., Fonseca, R., Dos Santos, D. M., Cupido, L., Mourão, A., Smoot, G. F., \& Tello, C. 2006, in New Worlds in Astroparticle Physics: Proceedings of the Fifth International Workshop, ed. A. M. Mourão, M. Pimenta, R. Potting, \& P. M. Sá, 233-+

Barkats, D., et al. 2005, Astrophys. J., Lett., 619, L127, astro-ph/0409380

Battistelli, E. S., Rebolo, R., Rubiño-Martín, J. A., Hildebrandt, S. R., Watson, R. A., Gutiérrez, C., \& Hoyland, R. J. 2006, Astrophys. J., Lett., 645, L141, arXiv:astro-ph/0603379

Baumann, D. et al. 2008a, ArXiv e-prints, 0811.3911

—. 2008b, ArXiv e-prints, 0811.3919

Beck, R. 2007, in EAS Publ. Ser. 23, Sky Polarisation at far-infrared to radio wavelengths: The Galactic Screen before the Cosmic Microwave Background, ed. F. Boulanger \& M.-A. MivilleDeschênes (EDP Sciences), 19

Bennett, C. L. et al. 2003, Astrophys. J., 583, 1 
Berdyugin, A., Piirola, V., \& Teerikorpi, P. 2004, Astron. Astrophys., 424, 873

Betoule, M., Pierpaoli, E., Delabrouille, J., Le Jeune, M., \& Cardoso, J.-F. 2008, in preparation for submission to Astronomy and Astrophysics

Bock, J. et al. 2006, ArXiv Astrophysies e-prints, arXiv:astro-ph/0604101

—. 2008, ArXiv e-prints, 0805.4207

Bottino, M., Banday, A. J., \& Maino, D. 2008, Mon. Not. R. Astron. Soc., 389, 1190

Cardoso, J.-F., Martin, M., Delabrouille, J., Betoule, M., \& Patanchon, G. 2008, ArXiv e-prints, 0803.1814

Casassus, S., Cabrera, G. F., Förster, F., Pearson, T. J., Readhead, A. C. S., \& Dickinson, C. 2006, Astrophys. J., 639, 951, arXiv:astro-ph/0511283

Chon, G., Challinor, A., Prunet, S., Hivon, E., \& Szapudi, I. 2004, Mon. Not. Roy. Astron. Soc., 350,914 , astro-ph/0303414

Christensen, N., Meyer, R., Knox, L., \& Luey, B. 2001, Classical and Quantum Gravity, 18, 2677

Chuss, D. T., Davidson, J. A., Dotson, J. L., Dowell, C. D., Hildebrand, R. H., Novak, G., \& Vaillancourt, J. E. 2003, Astrophys. J., 599, 1116

Crill, B. P. et al. 2008, ArXiv e-prints, 0807.1548

Curran, R. L., \& Chrysostomou, A. 2007, Mon. Not. R. Astron. Soc., 382, 699, arXiv:0709.0256

Davies, R. D., Dickinson, C., Banday, A. J., Jaffe, T. R., Górski, K. M., \& Davis, R. J. 2006, Mon. Not. R. Astron. Soc., 370, 1125, arXiv:astro-ph/0511384

Davis, L. J., \& Greenstein, J. L. 1951, Astrophys. J., 114, 206

de Oliveira-Costa, A., Kogut, A., Devlin, M. J., Netterfield, C. B., Page, L. A., \& Wollack, E. J. 1997, Astrophys. J., Lett., 482, L17+

de Oliveira-Costa, A., \& Tegmark, M. 2006, Phys. Rev. A, 74, 023005, arXiv:astro-ph/0603369

de Oliveira-Costa, A., et al. 2002, Astrophys. J., 567, 363

Delabrouille, J., \& Cardoso, J. . 2007, ArXiv Astrophysics e-prints, arXiv:astro-ph/0702198

Delabrouille, J., Cardoso, J.-F., \& Patanchon, G. 2003, Mon. Not. R. Astron. Soc., 346, 1089, arXiv:astro-ph/0211504

Dickinson, C., Davies, R. D., Bronfman, L., Casassus, S., Davis, R. J., Pearson, T. J., Readhead, A. C. S., \& Wilkinson, P. N. 2007, Mon. Not. R. Astron. Soc., 379, 297, arXiv:astro-ph/0702611

Dickinson, C., Davies, R. D., \& Davis, R. J. 2003, Mon. Not. R. Astron. Soc., 341, 369, arXiv:astro$\mathrm{ph} / 0302024$

Dobler, G., Draine, B. T., \& Finkbeiner, D. P. 2008, ArXiv e-prints, 0811.1040

Dobler, G., \& Finkbeiner, D. P. 2008a, Astrophys. J., 680, 1222, 0712.1038

—. 2008b, Astrophys. J., 680, 1235, 0712.2238

Dolginov, A. Z. 1972, APSS, 18, 337

Dolginov, A. Z., \& Mytrophanov, I. G. 1976, APSS, 43, 257

Dotson, J. L., Davidson, J., Dowell, C. D., Schleuning, D. A., \& Hildebrand, R. H. 2000, Astrophys. J., Suppl. Ser., 128, 335 
Dotson, J. L., Davidson, J. A., Dowell, C. D., Hildebrand, R. H., Kirby, L., \& Vaillancourt, J. E. 2009, ApJS, submitted

Draine, B. T., \& Fraisse, A. A. 2008, ArXiv e-prints, 0809.2094

Draine, B. T., \& Lazarian, A. 1998a, Astrophys. J., Lett., 494, L19

—. 1998b, Astrophys. J., 508, 157

—. 1999, Astrophys. J., 512, 740

Draine, B. T., \& Li, A. 2001, Astrophys. J., 551, 807, arXiv:astro-ph/0011318

Draine, B. T., \& Weingartner, J. C. 1996, Astrophys. J., 470, 551

—. 1997, Astrophys. J., 480, 633

Dunkley, J., Bucher, M., Ferreira, P. G., Moodley, K., \& Skordis, C. 2005, Mon. Not. R. Astron. Soc., 356, 925, arXiv:astro-ph/0405462

Dunkley, J. et al. 2008a, ArXiv e-prints, 803, 0803.0586

—. 2008b, ArXiv e-prints, 0811.4280

Eriksen, H. K. et al. 2006, Astrophys. J., 641, 665, arXiv:astro-ph/0508268

Eriksen, H. K., Hansen, F. K., Banday, A. J., Górski, K. M., \& Lilje, P. B. 2004, Astrophys. J., 605, 14

Eriksen, H. K. et al. 2007a, Astrophys. J., 656, 641, arXiv:astro-ph/0606088

Eriksen, H. K., Jewell, J. B., Dickinson, C., Banday, A. J., Gorski, K. M., \& Lawrence, C. R. 2007b, ArXiv e-prints, 709, 0709.1058

Finkbeiner, D. P. 2003, Astrophys. J., Suppl. Ser., 146, 407, accepted (astro-ph/0301558)

—. 2004, Astrophys. J., 614, 186

Finkbeiner, D. P., Davis, M., \& Schlegel, D. J. 1999, Astrophys. J., 524, 867, astro-ph/9905128

Finkbeiner, D. P., Langston, G. I., \& Minter, A. H. 2004, Astrophys. J., 617, 350

Fixsen, D. J., Dwek, E., Mather, J. C., Bennett, C. L., \& Shafer, R. A. 1998, Astrophys. J., 508, 123, arXiv:astro-ph/9803021

Fosalba, P., Lazarian, A., Prunet, S., \& Tauber, J. A. 2002, Astrophys. J., 564, 762

Fraisse, A. A. et al. 2008, ArXiv e-prints, 0811.3920

Gold, B., et al. 2008, Astrophys. J., Suppl. Ser.

Gold, T. 1952, Mon. Not. R. Astron. Soc., 112, 215

Halverson, N. W. et al. 2002, Astrophys. J., 568, 38

Han, J. L. 2007, in IAU Symposium, Vol. 242, IAU Symposium, 55-63

Han, J.-L., Manchester, R. N., Lyne, A. G., Qiao, G. J., \& van Straten, W. 2006, Astrophys. J., accepted (astro-ph/0601357)

Han, J. L., \& Zhang, J. S. 2007, Astron. Astrophys., 464, 609, arXiv:astro-ph/0611213

Haslam, C. G. T., Klein, U., Salter, C. J., Stoffel, H., Wilson, W. E., Cleary, M. N., Cooke, D. J., \& Thomasson, P. 1981, Astron. Astrophys., 100, 209 
Heiles, C. 1996, Astrophys. J., 462, 316

- 2000, Astron. J., 119, 923

Hildebrand, R., \& Kirby, L. 2004, in ASP Conf. Ser. 309, Astrophysics of Dust, ed. A. N. Witt, G. C. Clayton, \& B. T. Draine (San Francisco: ASP), 515

Hildebrand, R. H., Dotson, J. L., Dowell, C. D., Schleuning, D. A., \& Vaillancourt, J. E. 1999, Astrophys. J., 516, 834

Hildebrand, R. H., \& Dragovan, M. 1995, Astrophys. J., 450, 663

Hildebrandt, S. R., Rebolo, R., Rubiño-Martín, J. A., Watson, R. A., Gutiérrez, C. M., Hoyland, R. J., \& Battistelli, E. S. 2007, Mon. Not. R. Astron. Soc., 382, 594, 0706.1873

Hinshaw, G. et al. 2007, Astrophys. J., Suppl. Ser., 170, 288, arXiv:astro-ph/0603451

—. 2003, Astrophys. J., Suppl. Ser., 148, 135

- 2008, ArXiv e-prints, 803, 0803.0732

Hoang, T., \& Lazarian, A. 2008, Mon. Not. R. Astron. Soc., 388, 117, 0707.3645

Hyvarinen, A. 1999, in Neural Processing Letters, 1-5

Jewell, J., Levin, S., \& Anderson, C. H. 2004, Astrophys. J., 609, 1, arXiv:astro-ph/0209560

Keating, B. G., Timbie, P. T., Polnarev, A., \& Steinberger, J. 1998, Astrophys. J., 495, 580

Kogut, A., Banday, A. J., Bennett, C. L., Górski, K. M., Hinshaw, G., \& Reach, W. T. 1996a, Astrophys. J., 460, 1

Kogut, A., Banday, A. J., Bennett, C. L., Górski, K. M., Hinshaw, G., Smoot, G. F., \& Wright, E. I. 1996b, Astrophys. J., Lett., 464, L5

Kogut, A. et al. 2007, Astrophys. J., 665, 355, arXiv:0704.3991

Komatsu, E. et al. 2008, ArXiv e-prints, 803, 0803.0547

Kovac, J. M., Leitch, E. M., Pryke, C., Carlstrom, J. E., Halverson, N. W., \& Holzapfel, W. L. 2002, Nature, 420,772

La Porta, L., Burigana, C., Reich, W., \& Reich, P. 2008, Astron. Astrophys., 479, 641, 0801.0547

Larson, D. L., Eriksen, H. K., Wandelt, B. D., Górski, K. M., Huey, G., Jewell, J. B., \& O'Dwyer, I. J. 2007, Astrophys. J., 656, 653, arXiv:astro-ph/0608007

Lazarian, A. 2003, J. Quant. Spectros. Radiat. Transfer, 79, 881, arXiv:astro-ph/0208487

—. 2007, J. Quant. Spectros. Radiat. Transfer, 106, 225, arXiv:astro-ph/0208487

Lazarian, A., \& Draine, B. T. 2000, Astrophys. J., Lett., 536, L15

Lazarian, A., \& Finkbeiner, D. 2003, New Astronomy Review, 47, 1107

Lazarian, A., \& Hoang, T. 2007, Mon. Not. R. Astron. Soc., 378, 910, 0707.0886

Leach, S. M. et al. 2008, ArXiv e-prints, 0805.0269

Lee, A. T., et al. 2001, Astrophys. J., Lett., 561, L1, astro-ph/0104459

Leitch, E. M. et al. 2002, Nature, 420, 763

Leitch, E. M., Readhead, A. C. S., Pearson, T. J., \& Myers, S. T. 1997, Astrophys. J., Lett., 486, L23 
Leonardi, R. et al. 2007, ArXiv e-prints, 0704.0810

Lewis, A., \& Bridle, S. 2002, Phys. Rev. A, 66, 103511

Magalhães, A. M. et al. 2005, in Astronomical Society of the Pacific Conference Series, Vol. 343, Astronomical Polarimetry: Current Status and Future Directions, ed. A. Adamson, C. Aspin, C. Davis, \& T. Fujiyoshi, 305-+

Maino, D., Donzelli, S., Banday, A. J., Stivoli, F., \& Baccigalupi, C. 2007, Mon. Not. R. Astron. Soc., 374,1207

Maino, D. et al. 2002, Mon. Not. R. Astron. Soc., 334, 53, arXiv:astro-ph/0108362

Martin, P. G. 2007, in EAS Publ. Ser. 23, Sky Polarisation at far-infrared to radio wavelengths: The Galactic Screen before the Cosmic Microwave Background, ed. F. Boulanger \& M.-A. MivilleDeschênes (EDP Sciences), 165

Mason, B., Robishaw, T., \& Finkbeiner, D. 2008, in Astronomical Society of the Pacific Conference Series, Vol. 395, Frontiers of Astrophysics: A Celebration of NRAO's 50th Anniversary, ed. A. H. Bridle, J. J. Condon, \& G. C. Hunt, 373-+

Mathis, J. S., Rumpl, W., \& Nordsieck, K. H. 1977, Astrophys. J., 217, 425

Matthews, B. C., McPhee, C., Fissel, L., \& Curran, R. 2008, Astrophys. J., Suppl. Ser., submitted

Matthews, B. C., Wilson, C. D., \& Fiege, J. D. 2001, Astrophys. J., 562, 400

Metropolis, N., Rosenbluth, A. W., \& Rosenbluth, M. N. and Teller, A. H. 1953, J. Chem. Phys., 21,1087

Miller, A. D., et al. 1999, Astrophys. J., Lett., 524, L1, astro-ph/9906421

Miville-Deschenes, M. ., Ysard, N., Lavabre, A., Ponthieu, N., Macias-Perez, J. F., Aumont, J., \& Bernard, J. P. 2008, ArXiv e-prints, 802, 0802.3345

Montroy, T. E. et al. 2006, Astrophys. J., 647, 813, arXiv:astro-ph/0507514

Netterfield, C. B., et al. 2002, Astrophys. J., 571, 604, astro-ph/0104460

Neugebauer, G. et al. 1984, Astrophys. J., Lett., 278, L1

O’Dwyer, I. J., et al. 2004, Astrophys. J., 617, L99, astro-ph/0407027

Oxley, P. et al. 2004, in Presented at the Society of Photo-Optical Instrumentation Engineers (SPIE) Conference, Vol. 5543, Society of Photo-Optical Instrumentation Engineers (SPIE) Conference Series, ed. M. Strojnik, 320-331

P. Ade et al. 2007, ArXiv e-prints, 705, 0705.2359

Page, L. et al. 2007, Astrophys. J., Suppl. Ser., 170, 335, arXiv:astro-ph/0603450

Patanchon, G., Cardoso, J.-F., Delabrouille, J., \& Vielva, P. 2005, Mon. Not. R. Astron. Soc., 364, 1185, arXiv:astro-ph/0410280

Pearson, T. J. et al. 2003, Astrophys. J., 591, 556

Pereyra, A., \& Magalhães, A. M. 2004, Astrophys. J., 603, 584

—. 2007, Astrophys. J., 662, 1014, arXiv:astro-ph/0702550

Ponthieu, N., et al. 2005, Astron. \& Astro,, 607, 655, astro-ph/0501427

Prunet, S., \& Lazarian, A. 1999, in Microwave Foregrounds. Sloan Summit, Institute for Advanced 
Study, Princeton, New Jersey 14-15 November 1998. Eds: A. Rde Oliveira-Costa and M. Tegmark., Vol. 181 (Astronomical Society of the Pacific), 113, astro-ph/9902314

Prunet, S., Sethi, S. K., Bouchet, F. R., \& Miville-Deschenes, M.-A. 1998, Astron. Astrophys., 339, 187

Pryke, C. et al. 2008, ArXiv e-prints, 0805.1944

Readhead, A. C. S. et al. 2004, Astrophys. J., 609, 498

Roberge, W. G. 2004, in ASP Conf. Ser. 309, Astrophysics of Dust, ed. A. N. Witt, G. C. Clayton, $\&$ B. T. Draine, 467

Rybicki, G. B., \& Lightman, A. 1979, Radiative Processes in Astrophysics (Wiley \& Sons: New York)

Samtleben, D. 2008, ArXiv e-prints, 0806.4334

Savage, B. D., \& Sembach, K. R. 1996, ARAA, 34, 279

Schlegel, D. J., Finkbeiner, D. P., \& Davis, M. 1998, Astrophys. J., 500, 525, astro-ph/9710327

Schleuning, D. A. 1998, Astrophys. J., 493, 811

Schleuning, D. A., Vaillancourt, J. E., Hildebrand, R. H., Dowell, C. D., Novak, G., Dotson, J. L., \& Davidson, J. A. 2000, Astrophys. J., 535, 913

Scott, P. F. et al. 2003, Mon. Not. R. Astron. Soc., 341, 1076, astro-ph/0205380

Sembach, K. R., \& Savage, B. D. 1996, Astrophys. J., 457, 211

Smith, K. M. et al. 2008, ArXiv e-prints, 0811.3916

Spergel, D. N. et al. 2007, Astrophys. J., Suppl. Ser., 170, 377, arXiv:astro-ph/0603449

—. 2003, Astrophys. J., Suppl. Ser., 148, 175

Stompor, R., Leach, S., Stivoli, F., \& Baccigalupi, C. 2008, ArXiv e-prints, 0804.2645

Strong, A. W., Moskalenko, I. V., \& Ptuskin, V. S. 2007, Annual Review of Nuclear and Particle Science, 57, 285, arXiv:astro-ph/0701517

Takahashi, Y. D. et al. 2008, in Proc. SPIE 7020, Millimeter and Submillimeter Detectors and Instrumentation, ed. W. D. Duncan, W. S. Holland, S. Withington, \& J. Zmuidzinas, 7020-1D

Taylor, A. C. 2006, New Astronomy Review, 50, 993, arXiv:astro-ph/0610716

Taylor, A. R. et al. 2003, Astron. J., 125, 3145

Tegmark, M., de Oliveira-Costa, A., \& Hamilton, A. J. 2003, Phys. Rev. A, 68, 123523

Tegmark, M., \& Efstathiou, G. 1996, MNRAS, 281, 1297, astro-ph/9507009

The Planck Collaboration. 2006, ArXiv Astrophysics e-prints, arXiv:astro-ph/0604069

Tristram, M. et al. 2005, Astron. Astrophys., 436, 785, arXiv:astro-ph/0411633

Uyaniker, B., Landecker, T. L., Gray, A. D., \& Kothes, R. 2003, Astrophys. J., 585, 785, arXiv:astro$\mathrm{ph} / 0211436$

Vaillancourt, J. E. 2002, Astrophys. J., Suppl. Ser., 142, 53

Vaillancourt, J. E. 2007, in EAS Publ. Ser. 23, Sky Polarisation at far-infrared to radio wavelengths: The Galactic Screen before the Cosmic Microwave Background, ed. F. Boulanger \& M.-A. Miville- 
Deschênes (EDP Sciences), 147

Verde, L., Peiris, H., \& Jimenez, R. 2006, JCAP, 019, astro-ph/0506036

Wandelt, B. D., Larson, D. L., \& Lakshminarayanan, A. 2004, Phys. Rev. A, 70, 083511, arXiv:astro$\mathrm{ph} / 0310080$

Watson, R. A., Rebolo, R., Rubiño-Martín, J. A., Hildebrandt, S., Gutiérrez, C. M., FernándezCerezo, S., Hoyland, R. J., \& Battistelli, E. S. 2005, Astrophys. J., Lett., 624, L89

Whittet, D. C. B. 2003, Dust in the Galactic Environment, 2nd edn., Series on Astronomy and Astrophysics (Philadelphia: Institute of Physics Publishing)

Whittet, D. C. B. 2004, in ASP Conf. Ser. 309, Astrophysics of Dust, ed. A. N. Witt, G. C. Clayton, $\&$ B. T. Draine, 65

Whittet, D. C. B., Gerakines, P. A., Hough, J. H., \& Shenoy, S. S. 2001, Astrophys. J., 547, 872

Wolleben, M., Landecker, T. L., Reich, W., \& Wielebinski, R. 2006, Astron. Astrophys., 448, 411, arXiv:astro-ph/0510456

Zaldarriaga, M. et al. 2008, ArXiv e-prints, 0811.3918

Zweibel, E. G., \& Heiles, C. 1997, Nature, 385, 131 